\title{
The Complexity of Obstruction-Free Implementations
}

\author{
Hagit Attiya \\ Technion \\ and \\ Rachid Guerraoui \\ EPFL \\ and \\ Danny Hendler \\ Ben-Gurion University \\ and \\ Petr Kuznetsov \\ TU Berlin/Deutsche Telekom Laboratories
}

Obstruction-free implementations of concurrent objects are optimized for the common case where there is no step contention, and were recently advocated as a solution to the costs associated with synchronization without locks. In this paper, we study this claim and this goes through precisely defining the notions of obstruction-freedom and step contention. We consider several classes of obstruction-free implementations, present corresponding generic object implementations, and prove lower bounds on their complexity. Viewed collectively, our results establish that the worstcase operation time complexity of obstruction-free implementations is high, even in the absence of step contention. We also show that lock-based implementations are not subject to some of the time-complexity lower bounds we present.

Categories and Subject Descriptors: D.1.3 [Software]: Programming Techniques-Concurrent programming; F.1.2 [Theory of Computation]: Computation by Abstract Devices-Modes of Computation; F.2 [Theory of Computation]: Analysis of Algorithms and Problem Complexity

General Terms: Algorithms, Theory

Additional Key Words and Phrases: shared memory, solo-fast implementations, perturbable objects, step contention, memory contention, lower bounds

This paper combines results that appeared, in preliminary form, in [Attiya et al. 2005; Attiya et al. 2006].

H. Attiya is with the Department of Computer Science, Technion; supported by the Israel Science Foundation (grant number 953/06).

R. Guerraoui is with the School of Computer and Communication Sciences, EPFL; part of this work was done while the author visited the Computer Science and Artificial Intelligence Laboratory, MIT.

D. Hendler is with the Department of Computer Science, Ben-Gurion university; part of this work was done while the author was a post-doctoral fellow in the Faculty of Industrial Engineering and Management, Technion; supported by the Israel Science Foundation (grant number 1344/06).

P. Kuznetsov is at the Technische Universität Berlin/Deutsche Telekom Laboratories, part of this work was done while the author was at the School of Computer and Communication Sciences, EPFL, and Max Planck Institute for Software Systems.

Permission to make digital/hard copy of all or part of this material without fee for personal or classroom use provided that the copies are not made or distributed for profit or commercial advantage, the ACM copyright/server notice, the title of the publication, and its date appear, and notice is given that copying is by permission of the ACM, Inc. To copy otherwise, to republish, to post on servers, or to redistribute to lists requires prior specific permission and/or a fee.

(C) 2009 ACM 0004-5411/2009/0100-0001 $\$ 5.00$ 


\section{INTRODUCTION}

Major chip manufacturers are changing their focus from improving the speed of individual processors to increasing parallel processing capabilities. With multicore and multi-processor systems becoming commonplace, most computer programs will be concurrent, in some form or another. A major challenge for the theory of distributed computing is to devise efficient algorithms to cope with concurrent accesses to shared data objects. The operations of these objects would typically be implemented in software, out of more elementary primitives exported by base objects.

Traditional locking-based techniques are known to scale poorly and may induce deadlock and fault-tolerance issues. In contrast, implementations of shared objects that do not use locks require processes to coordinate without relying on mutual exclusion, thus avoiding the inherent problems of locking. The safety property typically required from these implementations is linearizability [Herlihy 1991; Herlihy and Wing 1990]; roughly, every operation on the object should appear to take effect instantaneously between its invocation and response.

Algorithms that avoid the use of locks are often, however, inefficient. Not surprisingly, the source of the inefficiency stems from handling contended situations. In fact, it is often argued [Herlihy et al. 2003; Herlihy et al. 2003] that, although contention should indeed be expected and handled appropriately, it is rare or can be made so through the use of appropriate mechanisms for managing contention. It is thus tempting to figure out the extent to which shared object implementations can exploit this rarity. In short, this would consist in devising shared object implementations that plan for the worst (high contention) and hope for the best (no contention). A natural way to explore this approach is to look for implementations that always preserve safety (linearizability) but have their termination depend on the absence of contention, i.e., ensuring (or expediting) termination when there is no contention. This paper studies the inherent complexity of such implementations.

In these implementations, fast termination is expected in the absence of step contention. When there is step contention, termination might not even be ensured. An operation may return control to a higher-level entity, called a client, which might decide if and when to retry the operation and may elect to use more sophisticated base objects. The term obstruction-free [Herlihy et al. 2003] has been used to coin these implementations. Informally, an implementation is called obstruction-free, "if it guarantees progress for every thread that eventually executes in isolation. Even though other threads may be in the midst of executing operations, ..." [Herlihy et al. 2003, Page 522].

Nothing was known on the inherent complexity of such implementations and, in fact, we are not aware of any attempt to precisely define them.

The first contribution of this paper is to precisely characterize obstruction-free implementations and this, itself, goes first through formally capturing the very notion of contention. We provide the first formal definition of the concept of step contention, according to which processes should only be considered contending on the implemented shared object if they concurrently apply primitive operations on its base objects. The absence of step contention allows scenarios where other processes have pending operations on the same implemented object but are not accessing the

Journal of the ACM, Vol. V, No. N, April 2009. 


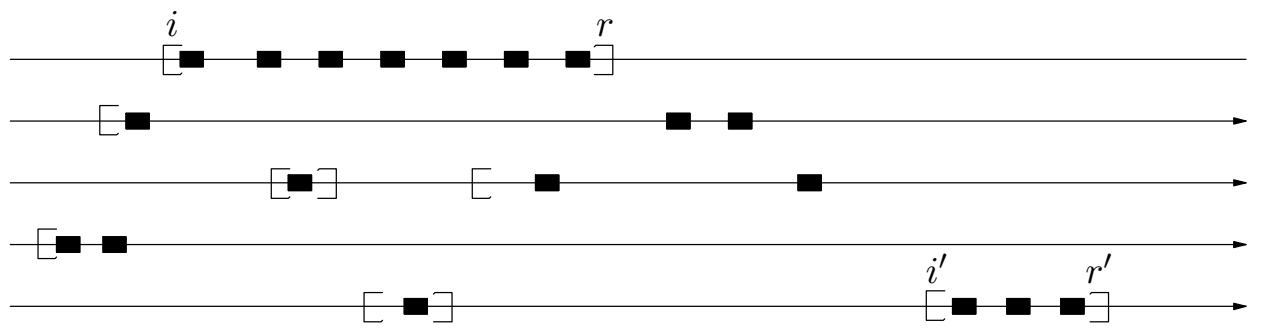

Fig. 1. Operation $[i, r]$ has interval contention 5 , point contention 4, and step contention 3; operation $\left[i^{\prime}, r^{\prime}\right]$ has interval and point contention 4 , and step contention $1\left(\left[i^{\prime}, r^{\prime}\right]\right.$ is step-contention free). Square brackets denote invocations and responses, while solid intervals denote steps on base objects.

base objects of the implementation. This is fundamentally different from alternative contention metrics: point contention [Attiya and Fouren 2003] and interval contention [Afek et al. 2002], both counting also failed or swapped-out processes. (See the scenario presented in Figure 1.)

Unlike interval contention or point contention, a process encounters step contention on an implemented object only if other processes concurrently apply primitive operations on its base objects. Clearly, implementations that would guarantee termination only in the absence of interval (or point) contention might not be robust: failed (or swapped-out) processes can be used as an excuse for other processes to never terminate. An implementation might not tolerate a single process failure while being obstruction-free with respect to interval contention for instance. In short, obstruction-freedom with respect to point or interval contention do not preclude the use of locking. Our goal is to study the inherent complexity of implementations that are robust (in particular those who guarantee global progress and thus preclude locking), yet that exploit the rarity of step contention.

If a client application is not willing to wait indefinitely for operation completion, it may be beneficial for an obstruction-free implementation to return a fail indication to the client in the face of step contention. By doing that, the implementation enables the client to choose whether to re-invoke the same operation or to invoke another one. We show however, by reduction to the (wait-free) consensus impossibility [?; Loui and Abu-Amara 1987], that there is an inherent uncertainty as to whether the operation could have had an effect on the object or not (Theorem 1). When the implementation uses primitives that cannot solve consensus, e.g., reads and writes, this implies that the implementation cannot be guaranteed to return either a legal response or a fail indication. ${ }^{1}$

Several classes of obstruction-free implementations can be considered, and these differ based on how step contention is handled once detected and what base objects are available. We precisely define each class, we present corresponding generic object implementations and we give lower bounds on their complexity.

\footnotetext{
${ }^{1}$ The interface between the implementation and the client can be enriched so that an implementation sometimes return a special pause value, indicating that the client should re-invoke the same operation (see Appendix A). The notion of linearizability can be extended accordingly so as to accommodate failed operations and re-invocations of paused operations.
} 
To measure the performance of obstruction-free implementations, we study their step contention-free complexity: this is the number of steps taken by a process running alone, until it returns a value. By focusing on the cost of the implementations in executions where no process encounters step contention, we capture here the complexity of obstruction-free implementations in situations where obstruction-freedom is expected to be especially beneficial.

We first study obstruction-free implementations that are restricted to use only overwriting or trivial primitives (e.g., write, swap and read). We present a generic obstruction-free object implementation which has a linear step contention-free complexity and uses a linear number of read/write objects (Theorem 10). We show that these complexities are asymptotically optimal, by noticing that they are subject to the lower bound of Jayanti et al. [2000] (Observation 1). This implies that obstruction-free implementations of perturbable objects, e.g., fetch\&add, modulo- $b$ counter (for $b \geq 2 n$ ), $b$-valued compare\&swap (for $b \geq n$ ), and LL/SC bits, using overwriting or trivial primitives must have $\Omega(n)$ step contention-free complexity and must use $\Omega(n)$ base objects.

We then study a class of obstruction-free implementations that are restricted to use simple primitives when there is no step contention, but might use more powerful primitives when there is. More specifically, these are wait-free linearizable object implementations that use only (cheap) overwriting or trivial primitives when there is no step contention, but may fall back on more powerful (and expensive) primitives like compare\&swap, when step contention occurs. Such implementations are also called solo-fast in the sense that termination is faster when there is no step contention. We give a solo-fast implementation of consensus, which we believe is interesting in its own right, and derive a generic solo-fast implementation of any object type (Theorem 2). This generic implementation has a linear step contention-free complexity. We prove that such implementations must indeed have non-constant step and space complexity, i.e., they are also inherently expensive. Specifically, an $n$-process solo-fast implementation of a perturbable object, using only overwriting or trivial primitives in step-contention free executions, must have $\Omega(n)$ space complexity (Theorem 4) and $\Omega(\log n)$ step contention-free complexity (Theorem 5).

We finally consider obstruction-free implementations that use powerful primitives even when there is no step contention. We prove that, for any obstructionfree implementation of binary consensus, there exist executions where no process is aware of step contention, in which either some process accesses $\sqrt{n}$ distinct base objects while performing a single operation, or some process incurs $\sqrt{n}$ memory stalls [Dwork et al. 1997] (Theorem 6). The stalls metric captures the fact that when process $p$ applies a nontrivial primitive to base object $r$ right after $k$ distinct processes other than $p$ apply nontrivial primitives to $r$, then $p$ suffers a delay of length proportional to $k$. This result holds also for implementations of any perturbable object (Theorem 7). Since these results are obtained in executions where none of the processes is aware of step contention, they hold for any obstructionfree implementation of these objects, regardless of how processes behave if they become aware of step contention. We also prove that, in any obstruction-free implementation of a perturbable object in which processes are not allowed to fail their

Journal of the ACM, Vol. V, No. N, April 2009. 
operations, the number of memory stalls incurred by some process that is unaware of step contention is $\Omega(n)$ (Theorem 9). We show that lock-based implementations of perturbable objects are not subject to these bounds, thus establishing a separation between the worst-case operation time-complexity of obstruction-free and lock-based implementations.

To summarize, the contributions of this paper are the following. We define the notion of step contention and use it to define obstruction-free implementations (Section 2), and show that a obstruction-free consensus implementation from registers cannot return just legal values or fail (Section 3). We then study the complexity of different classes of obstruction-free implementations that use strong synchronization primitives only in the presence of step contention (Section 4) as well those that can use these primitives even in the absence of step contention (Section 5).

\section{MODEL}

We use a standard model of an asynchronous shared memory system, in which a set $\mathbf{P}$ of $n>1$ processes $p_{1}, \ldots, p_{n}$ communicate through shared objects.

\subsection{Objects and Implementations}

Every object has a type that is defined by a quadruple $(Q, O, R, \Delta)$, where $Q$ is a set of states, $O$ is a set of invocations, $R$ is a set of responses, and $\Delta \subseteq Q \times O \times Q \times R$ is a relation, known as the sequential specification of the type. This relation specifies all the sequences of invocation-response pairs allowed by the type.

For example, the compare\&swap object is accessed by a $C \mathscr{E} S\left(r_{1}, r_{2}, m\right)$ operation; the operation compares the value in memory location $m$ with the content of local variable $r_{1}$, and if equal, writes the value of $r_{2}$ to $m$. The operation returns true if the compared values are equal (and the swap succeeds); otherwise, it returns false. The sequential specification of the compareßङswap type includes all sequences of $C \mathscr{B} S$ operations that obey this rule. We assume that a compareßsswap object also supports a read operation.

Another important example is the consensus object. Processes invoke a propose operation on the consensus object with an argument from some domain $V$. Processes need to agree on a single argument value. More formally, the sequential specification of consensus consists of all sequences of propose operations such that (1) all operations return the same value, and (2) the returned value is the argument of some propose operation.

To implement a (high-level) object from a set $\mathbf{B}$ of shared base objects, processes follow an algorithm, which is a collection of deterministic state machines, one for each process. The algorithm also assigns initial values to the base objects. To avoid confusion, we call operations on the base objects primitives, and reserve the term operations for the objects being implemented. We also say that an operation of an implemented object is performed and that a primitive is applied to a base object. No bound is assumed on the size of a base object (i.e., the number of distinct values the object can take).

We say that a primitive is nontrivial if it may change the value of the base object to which it is applied, e.g., a write or a read-modify-write, and trivial otherwise., e.g., a read. Let $o$ be an object that supports two primitives $f$ and $f^{\prime}$. Following Fich et al. [1998], we say that $f$ overwrites $f^{\prime}$ on $o$, if starting from any value $v$ of $o$, 
applying $f^{\prime}$ and then $f$ results in the same value as applying just $f$, using the same input parameters (if any) in both cases. A set of primitives is called historyless if all the nontrivial primitives in the set overwrite each other; we also require that each such primitive overwrite itself. A set that includes the write and swap primitives is an example of a historyless set of primitives.

In an operation instance $\Phi=\left(x, O p, p_{i}\right.$, args $)$, process $p_{i}$ performs the operation $O p$, with arguments args, on object $x$.

\subsection{Configurations, Executions and Histories}

A configuration specifies the value of each base object and the state of each process. In an initial configuration, all base objects have their initial values and all processes are in their initial states. A configuration is quiescent if no process is in the middle of performing an operation instance.

When receiving an invocation (to the high-level object), process $p_{i}$ takes steps according to its state machine. Each step of $p_{i}$ consists of some local computation and one shared memory event, which is a primitive applied to a base object. After each step, $p_{i}$ possibly changes its local state according to its state machine and possibly returns a response on the pending high-level operation. An event is nontrivial if it is an application of a nontrivial primitive.

An execution fragment is a (possibly infinite) sequence of events, that result from interleaving the steps taken by processes, according to their state machines. An execution is an execution fragment that starts from an initial configuration. For any finite execution fragment $\alpha$ and any execution fragment $\alpha^{\prime}$, the execution fragment $\alpha \alpha^{\prime}$ is the concatenation of $\alpha$ and $\alpha^{\prime}$; in this case $\alpha^{\prime}$ is called an extension of $\alpha$. An execution $\alpha$ is $Q$-free, for a non-empty set of processes $Q \subset \mathbf{P}$, if no events in $\alpha$ is applied by a process in $Q$. If $Q=\{q\}$, we say that $\alpha$ is $q$-free instead of $Q$-free.

If the last event of an operation instance $\Phi$ has been applied in an execution $\alpha$, we say that $\Phi$ completes in $\alpha$ and that $\Phi$ returns a response in $\alpha$.

In an infinite execution, a process is correct if it takes an infinite number of steps or it has no pending operation; otherwise, it is faulty.

We denote by $\alpha \mid p$ the subsequence of events of execution $\alpha$ that are applied by process $p$. Two executions are indistinguishable to a process $p$, if $p$ applies exactly the same sequence of events and gets the same responses from these events in both executions.

Every execution $\alpha$ induces a history $H(\alpha)$ that includes only the invocations and responses of the high-level operations. Each invocation or response is associated with a single process and a single object. A local history of process $p_{j}$ in $H, H \mid j$, is the subsequence of $H$ containing only events of $p_{j}$. Similarly, $H \mid x$ is the subsequence of $H$ of operations on an object $x$.

A response matches an invocation if they are associated with the same process and the same object. A local history is well-formed if it is a sequence of matching invocation-response pairs, except perhaps for the last invocation in a finite local history. A history $H$ is well-formed if every local history in $H$ is well-formed.

A pair of consecutive invocation and matching response $[i, r]$ is called a complete operation, and we say that $i$ returns $r$. An invocation $i$ without a matching response (in a given history $H$ ) is a pending (in $H$ ) operation; a completion of a pending operation (i.e., an invocation) is the invocation followed by a matching response.

Journal of the ACM, Vol. V, No. N, April 2009. 
The fragment of $H$ (or $\alpha$, its corresponding execution) between the invocation $i$ and its matching response $r$ (if it exists) is the operation's interval.

\subsection{Properties of Implementations}

A history $H$ is sequential if every invocation is immediately followed by its matching response. A sequential history $H$ is legal if for every object $x, H \mid x$ is in the sequential specification of $x$.

Two different invocations $i$ and $i^{\prime}$ on the same object $x$ are concurrent in a history $H$, if $i$ and $i^{\prime}$ are both pending in some finite prefix of $H$. This implies that their intervals overlap. We say that two operations $[i, r]$ and $\left[i^{\prime}, r^{\prime}\right]$ (or $i^{\prime}$ if $i^{\prime}$ is pending) are non-concurrent if their intervals are non-overlapping: Either $r$ appears before $i^{\prime}$ in $H$, in which case we say that $[i, r]$ precedes $\left[i^{\prime}, r^{\prime}\right]$, or $r^{\prime}$ appears before $i$ in $H$, in which case we say that $[i, r]$ follows $\left[i^{\prime}, r^{\prime}\right]$.

Definition 1. A well-formed history $H$ satisfies extended linearizability [Herlihy 1991] (see also [Attiya and Welch 2004, Chapter 10]) if there is a permutation $H^{\prime}$ containing all the complete operations and completions of a subset of the pending operations in $H$, such that (1) $H^{\prime}$ is legal, and (2) $H^{\prime}$ respects the order of non-concurrent operations in $H . H^{\prime}$ is the linearization of $H$.

In accordance with the literature, we simply call this property linearizability below.

A process $p$ is active after execution $\alpha$ if $p$ is in the middle of performing some operation instance $\Phi$, i.e., $p$ has applied at least one event while performing $\Phi$ in $\alpha$, but $\Phi$ does not complete in $\alpha$. If $p$ is not active after $\alpha$, we say that $p$ is idle after $\alpha$.

\subsection{Step contention and obstruction-freedom}

The step contention of an execution fragment $\alpha$ is the number of processes that take steps in $\alpha$. We say that $\alpha$ is step-contention free for $p$ if the events of $\alpha \mid p$ are contiguous in $\alpha$. Also, $\alpha$ is step-contention free if $\alpha$ is step-contention free for all processes. An operation $\Phi$ is eventually step-contention free in $\alpha$, if either $\Phi$ completes in $\alpha$ or if there is a suffix of $\alpha$ that consists only of the events of $\Phi$; in other words, starting from some point in $\alpha, \Phi$ runs solo.

An implementation is called obstruction-free "if it guarantees progress for every thread that eventually executes in isolation. Even though other threads may be in the midst of executing operations, ..." [Herlihy et al. 2003, Page 522]. This requirement is actually equivalent to solo termination [Fich et al. 1998], and it echoes the liveness correctness conditions stated for Paxos-style consensus algorithms in the context of state-machine replication [Lamport 1998]. Using our terminology, this leads to the following definition.

DEFINITION 2. An implementation is obstruction-free if every operation that is eventually step-contention free eventually returns.

\section{OBSTRUCTION-FREE IMPLEMENTATIONS USING ONLY READS AND WRITES}

Obstruction-freedom is a very weak liveness condition, and it requires the operation to return only under very restricted conditions. In all other circumstances, we only 


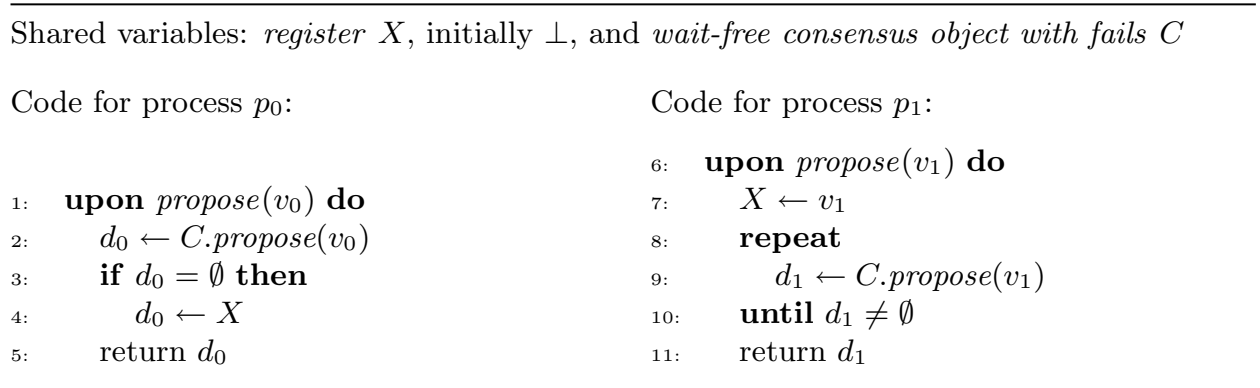

Fig. 2. Wait-free consensus from wait-free consensus with fails

require that an operation's response is legal (i.e., that it does not violate the safety properties of the implementation), if it returns a response at all.

We extend the interface of the implementation with a special fail value $\emptyset$. This value can only be returned when an operation cannot return a legal response and the implementation is certain that the failed operation is not going to influence the responses of concurrent or subsequent operations. When the client of the implementation (i.e., the higher-level entity that invokes operations on it and receives its responses) obtains a fail response, it is allowed to abort its current operation and invoke any other operation instead. This enables the client to avoid waiting indefinitely for the operation to complete in high step contention scenarios.

For this purpose, we add to the set $R$ of object responses a special fail value $\emptyset \notin R$. The definition of linearizability is also extended so that invocations returning fail are removed from the linearized history. To rule out trivial solutions, we require implementations to be valid: $\emptyset$ can only be returned if the corresponding operation is not step-contention free.

Note that allowing an implementation to return fail responses in case of step contention weakens the requirements from the implementation, and can potentially allow us to devise implementations that are impossible to devise otherwise. For instance, we could presumably implement a wait-free two-process consensus object from registers that is allowed to return $\emptyset$ in case of step contention to indicate that an operation did not take effect. We show, however, that this is impossible.

THEOREM 1. There is no wait-free valid implementation of consensus with fails from registers.

Proof. Suppose by way of contradiction that such an implementation exists. We show that it is then possible to implement wait-free consensus for two processes, $p_{0}$ and $p_{1}$, using one such consensus object, denoted $C$, and one register $X$, that can be written by $p_{1}$ and read by $p_{0}$. But this contradicts [Loui and Abu-Amara 1987].

The algorithm, described in Figure 2, is asymmetric: Process $p_{0}$ invokes a propose operation on $C$. If a non- $\emptyset$ value $v$ is returned, $p_{0}$ decides on $v$; otherwise, $p_{0}$ decides on the value it reads from the shared register $X$. Process $p_{1}$ starts by writing its value to $X$; then, $p_{1}$ repeatedly proposes in $C$, until it obtains a non- $\emptyset$ value $v$, and decides on it.

To see why a value returned has been proposed, note that the algorithm returns either a non- $\emptyset$ value obtained from the embedded consensus object $C$, or a value

Journal of the ACM, Vol. V, No. N, April 2009. 
read from $X$. In the first case, from the validity property of $C$, the value has been proposed. Otherwise, $p_{0}$ obtains a $\emptyset$ value from $C$. This can only occur if $p_{0}$ encounters step contention because of the steps of $p_{1}$. In this case, it is guaranteed that $p_{1}$ has previously written its input to $X$, which is the value $p_{0}$ decides on.

Clearly, the processes do not return different values when they both decide on a value returned from $C$; the only other case is when $p_{0}$ obtains $\emptyset$ from $C$, in which case, $p_{0}$ decides on $p_{1}$ 's input (which it reads from $X$ ); in this case, since $p_{0}$ 's propose on $C$ fails, $C$ can return only $p_{1}$ 's input, implying that $p_{1}$ can only decide on its own value.

Finally, to show that the algorithm is wait-free, note that $p_{0}$ performs a constant number of steps, and that $p_{1}$ eventually runs in the absence of step contention, and decides.

Theorem 1 implies that any implementation of consensus from registers has executions in which it does not terminate, even if it is allowed to return $\emptyset$ when step contention is encountered. On the other hand, it is straightforward to implement an obstruction-free consensus object from registers (see Appendix B). By replacing wait-free consensus objects in the universal construction of Herlihy [1991] with obstruction-free ones, we obtain a generic obstruction-free implementation of any object from registers.

The generic implementation uses $O(n)$ registers and ensures that every step contention-free operation terminates in $O(n)$ steps. In fact, linear time and space complexity is asymptotically optimal for implementations of a large class of perturbable objects. This class, originally defined by Jayanti et al. [2000], includes counters, fetch\&add and compare\&swap objects; see Definition 3 in Section 4.2 below. Jayanti et al. [2000] show that any implementation of a perturbable object that satisfies the solo termination property has an execution in which a solo operation (i.e., an operation that does not observe step contention) takes $n-1$ or more steps and accesses $n-1$ or more different base objects. Since any obstruction-free implementation ensures the solo termination property, we immediately have the next observation.

ObSERVATION 1. Let $A$ be an obstruction-free implementation of a pertubable object using only overwriting or trivial primitives. A has an execution in which a step-contention free operation takes $n-1$ or more steps and accesses $n-1$ or more different objects.

In Appendix A, we further extend the interface between a client and linearizable implementations with a special pause value. Pauses can only be returned if (1) there is step contention, and (2) the invoked operation might have taken effect (in a strict sense). We show that every object has an implementation from registers that provides the extended interface and always returns.

\section{OBSTRUCTION-FREE IMPLEMENTATIONS THAT ARE SOLO-FAST}

We call an implementation solo-fast if it only applies primitives from some historyless set of primitives in step-contention free executions but can apply additional primitives upon encountering step contention. In this section, we present a generic (wait-free) solo-fast implementation from registers and C\&S objects, and prove 
complexity lower bounds for solo-fast implementations.

\subsection{Generic Object Implementation}

Figure 3 presents a solo-fast consensus implementation. The algorithm proceeds in rounds (lines 13-24). Starting the algorithm, every process $p_{i}$ first computes in line 3 the smallest round $k_{i}$ in which a value can be fixed, i.e., returned in line 19 (we say that $p_{i}$ joins in round $k_{i}$ ). More precisely, $p_{i}$ joins in minimal round $k$ such that the registers $A_{1}, \ldots, A_{n}$ contain no values with timestamps higher than $k$ and no different values with timestamp $k$. In every round, starting from round $k_{i}, p_{i}$ tries to fix its current decision estimate $v_{i}$. The algorithm ensures that if no other process tries to fix concurrently any value in a higher round, or a different value in the current round, then the estimate is fixed. If $p_{i}$ is not able to fix the estimate in round $k$ (we say that $p_{i}$ loses round $k$ ), which can only happen when there is step contention, it updates the estimate using a $\mathrm{C} \& \mathrm{~S}$ operation and goes to round $k+1$. If no process joins in round $k+1$, then $p_{i}$ fixes its estimate in round $k+1$ (the use of C\&S ensures that no two processes that lost round $k$ try to fix different values in round $k+1$ ). We show that no process can join in round $n$ or later, and thus $p_{i}$ fixes its estimate in round $k \leq n$. The algorithm is solo-fast, since a process does not lose a round (and thus fall back to using a C\&S object) in the absence of step contention.

THEOREM 2. There is a solo-fast consensus implementation from registers and $C \mathscr{E} S$ objects, which takes $O(n)$ steps in the absence of step contention.

Proof. The algorithm clearly implies that only a proposed value can be returned.

We say that a process $p_{i}$ reaches round $k$ if it reaches line 13 with $k_{i}=k ; p_{i}$ participates in an execution if it reaches some round $k \geq 1$. A process $p_{i}$ joins in round $k$ if $k$ is the first round $p_{i}$ reaches. We say that the result $V$ of a collect of $A_{1}, \ldots, A_{n}$ or $B_{1}, \ldots, B_{n}$ is $k$-lost if $V$ contains values with timestamps higher than $k$ or two different values with timestamp $k$.

Claim 1. For all $k \geq 2$, if no process joins in round $k$ or later, then $A_{1}, \ldots, A_{n}$ contain no $\left(k^{\prime}, v^{\prime}\right)$ such that $k^{\prime}>k$ and no two $\left(k, v^{\prime}\right)$ and $\left(k, v^{\prime \prime}\right)$ such that $v^{\prime} \neq v^{\prime \prime}$.

Proof. If no process joins in round $k$ or later $(k \geq 2)$, then every process that reaches round $k$ previously completed round $k-1$ and invoked $C_{k-1} . C \mathscr{B} S\left(\perp, v_{i}\right)$ to update its current estimate (line 23). If $C_{k-1} . C \mathscr{E} S\left(\perp, v_{i}\right)$ fails, then the process adopts the value of $C_{k-1}$ as its current estimate. Otherwise, the process keeps its estimate unchanged. Thus, all processes that reach round $k$ have identical estimates. By the algorithm, a process can lose round $k$ only if it reads $\left(k^{\prime}, v^{\prime}\right)$ such that $k^{\prime}>k$. Since no process joins in round $k$ or later, this value can be written only by a process that previously lost round $k$. Thus, no process can lose round $k$ and write a value $\left(k^{\prime}, v^{\prime}\right)$ such that $k^{\prime}>k$ in $A_{1}, \ldots, A_{n}$.

Claim 2. Let $k \geq 2$ be the number of processes that participate in the algorithm. No process joins in round $k$ or later.

Proof. By induction on $k$. If there is only one participant, then it trivially joins and returns in round 1.

Journal of the ACM, Vol. V, No. N, April 2009. 




Fig. 3. An $n$-process solo-fast consensus: code for process $p_{i}$

Assume now that the claim holds for all $k^{\prime}, 2 \leq k^{\prime} \leq k$, and consider any execution with $k+1$ participants. Let $p_{i}$ be the last process that joins in that execution. Consider a prefix $\alpha$ of the execution in which $p_{i}$ just completed executing line 3 . Let $V$ be the result of the collect taken by $p_{i}$ in line 2 . Since $p_{i}$ does not write in $\alpha$, the other (at most $k$ ) participants cannot distinguish $\alpha$ from an execution with at most $k$ participants. By the induction hypothesis, no process joins in round $k$ or later in $\alpha$. By Claim 1, $V$ contains no $\left(k^{\prime}, v^{\prime}\right)$ such that $k^{\prime}>k$ and no two pairs $\left(k, v^{\prime}\right)$ and $\left(k, v^{\prime \prime}\right)$ such that $v^{\prime} \neq v^{\prime \prime}$. By the algorithm (line 3$), p_{i}$ joins in round $k$ or earlier and the claim follows.

Since there are at most $n$ participants, Claim 2 implies that no process joins in round $n$ or later. By Claim 1 , in any execution, $A_{1}, \ldots, A_{n}$ contain no $\left(k^{\prime}, v^{\prime}\right)$ such that $k^{\prime}>n$ and no two $\left(n, v^{\prime}\right)$ and $\left(n, v^{\prime \prime}\right)$ such that $v^{\prime} \neq v^{\prime \prime}$. It follows that any process that reaches round $n$ will pass the "if" clauses in lines 15 and 18 and return in line 19. Thus, every process decides in round $n$ or earlier.

Claim 3. Assume process $p_{i}$ returns $v$ in round $k . A_{1}, \ldots, A_{n}$ contain no $\left(k^{\prime}, v^{\prime}\right)$ such that $v^{\prime} \neq v$ and $k^{\prime} \geq k+1$ and $B_{1}, \ldots, B_{n}$ contain no $\left(k^{\prime}, v^{\prime}\right)$ such that $v^{\prime} \neq v$ 
and $k^{\prime} \geq k$.

Proof. Assume $p_{i}$ returns $v$ in round $k$. By the algorithm, it has previously written $(v, k)$ in $A_{i}$ and $B_{i}$. First, we observe that, for all $k$, if a process $p_{j}$ writes $\left(k^{\prime}, v^{\prime}\right)$ in $A_{j}$ and then $\left(k^{\prime}, v^{\prime \prime}\right)$ in $B_{j}$, then $v^{\prime}=v^{\prime \prime}$.

We proceed by induction on $m$, the number of processes that reach round $k+1$ or higher. Let $m=0$, i.e., no process reaches any round $k^{\prime} \geq k+1$. Trivially, no value with timestamp $k^{\prime} \geq k+1$ can be written in $A_{1}, \ldots, A_{n}$ and $B_{1}, \ldots, B_{n}$. To obtain a contradiction, assume that some process $p_{j}$ writes $\left(k, v^{\prime}\right)$ with $v^{\prime} \neq v$ in $B_{j}$. Thus, $p_{j}$ previously wrote $\left(k, v^{\prime}\right)$ in $A_{j}$, and then $p_{j}$ did not read $(k, v)$ in $A_{i}$ (otherwise, $p_{j}$ would not pass the "if" clause in line 15). Hence, $p_{i}$ has written $(k, v)$ in $A_{i}$ after $p_{j}$ has written $\left(k^{\prime}, v^{\prime}\right)$ in $A_{j}$. But then $p_{i}$ would necessarily read $\left(k, v^{\prime}\right)$ (or a value with a higher timestamp) in $A_{j}$ and would not pass the "if" clause in line 15 - a contradiction.

Now assume that, for some $m \geq 0$, the claim holds for every execution in which at most $m$ processes reach round $k+1$ or higher. Consider an execution in which $m+1$ processes reach round $k+1$ or higher. Let $p_{j}$ be any process to reach round $k+1$ or higher in that execution. By the algorithm $p_{j}$ collects $A_{1}, \ldots, A_{n}$ and joins in the minimal round $k^{\prime}$ such that the result of the collect operation is not $k^{\prime}$-lost, i.e., $p_{j}$ found no values with timestaps higher than $k^{\prime}$ and no different values with timestamps $k^{\prime}$. When process $p_{j}$ joins, at most $m$ processes reached round $k+1$ or higher. We consider the two possible cases:

(1) $p_{j}$ joins in round $k+1$ or higher.

By the algorithm, before joining, $p_{j}$ collects $A_{1}, \ldots, A_{n}$ in line 2 and then collects $B_{1}, \ldots, B_{n}$ in line 7 . Let $V$ and $V^{\prime}$ be the results of these two collects. Since $p_{j}$ joins in round $k+1$ or higher, $V$ is $k$-lost. But since $p_{i}$ returns $v$ in round $k$, it previously wrote $(k, v)$ in $B_{i}$ and then collected $A_{1}, \ldots, A_{n}$, so that the result $V^{\prime \prime}$ of the last collect is not $k$-lost. Thus, $p_{j}$ read $B_{i}$ in its collect of $B_{1}, \ldots, B_{n}$ after $p_{i}$ wrote $(k, v)$ in it. Otherwise, since the timestamps of values written in the same register grow monotoncally, $V^{\prime \prime}$ would also be $k$-lost, and $p_{i}$ would not be able to return in round $k$. Hence, $V^{\prime}$ contains $(k, v)$.

Further, by the induction hypothesis, $V$ contains no value $v^{\prime} \neq v$ timestamped with $k+1$ or higher and $V^{\prime}$ contains no value $v^{\prime} \neq v$ timestamped with $k$ or higher. Thus, if $p_{j}$ finds a value with timestamp $k^{\prime} \geq k+1$ in $V$, then this value is $v$, and $p_{i}$ adopts $v$ as its estimate (line 5). Otherwise, $p_{j}$ adopts the value with the highest timestamp in $V^{\prime}$ (line 9), and, since $(k, v) \in V^{\prime}$ and no value different from $v$ has timestamp $k+1$ or higher in $V^{\prime}, p_{j}$ adopts $v$.

Finally, in round $k^{\prime}, p_{j}$ writes $\left(k^{\prime}, v\right)$ in $A_{j}$.

(2) $p_{j}$ participates in round $k$.

Thus, in round $k, p_{j}$ collects $A_{1}, \ldots, A_{n}$ so that the result of the collect operation was $k$-lost (line 14 or line 17), and then collects $B_{1}, \ldots, B_{n}$ in line 20 . Let $V$ and $V^{\prime}$ be the results of these two collects. Again, since $p_{i}$ returns $v$ in round $k, p_{i}$ wrote $(k, v)$ in $B_{i}$ before $p_{j}$ read $B_{i}$ in its collect of $B_{1}, \ldots, B_{n}$. Hence, $V^{\prime}$ contains $(k, v)$. By the hypothesis, when $p_{j}$ completes the second collect operation, at most $m$ processes reached round $k+1$ or higher, and, thus, $V$ contains no value $v^{\prime} \neq v$ timestamped with $k^{\prime} \geq k+1$, and $V^{\prime}$ contains no value $v^{\prime} \neq v$ timestamped with $k^{\prime} \geq k$. By the algorithm, $p_{j}$ adopts the highest 
timestamped value in $V^{\prime}$ (if any) in line 22. Thus, any process that accesses the C\&S object $C_{k}$ previously adopted $v$ as its estimate. If $p_{j}$ fails when invoking $C_{k}, C \mathscr{E} S(\perp, v)$, then it reads $v$ from $C_{k}$. In both cases, when $p_{j}$ begins round $k+1, v_{j}=v$.

Finally, in round $k+1, p_{j}$ writes $(k+1, v)$ in $A_{j}$.

In both cases, when $p_{j}$ reaches its first round higher than $k$, it cannot write a value different from $v$, implying the inductive claim.

Let $k$ be the first round in which some process $p_{i}$ decides some value $v$. By the algorithm, if a process $p_{j}$ decides $v^{\prime}$ in a round $k^{\prime}$, then it previously wrote $\left(k^{\prime}, v^{\prime}\right)$ in $B_{j}$ (line 16). By Claim 3, no process $p_{j}$ writes $\left(k^{\prime}, v^{\prime}\right)$ such that $v^{\prime} \neq v$ and $k^{\prime} \geq k$. Thus, no process decides $v^{\prime} \neq v$, implying that processes do not decide on different values.

Finally, we need to show that the implementation is solo-fast. Assume that a process $p_{i}$ joins in round $k$. The only reason for $p_{i}$ to lose that round is to observe a value timestamped with $k^{\prime}>k$ or two different values timestamped with $k$ in $A_{1}, \ldots, A_{n}$ (line 15 or 18). But $p_{i}$ previously observed the opposite in line 3 and adopted the value timestamped with $k$ found in $A_{1}, \ldots, A_{n}$ (if any) in line 5 . Thus, $p_{i}$ can lose round $k$ only when some other process $p_{j}$ concurrently writes $\left(k^{\prime}, v^{\prime}\right)$ in $A_{j}$ such that $k^{\prime}>k \vee\left(k^{\prime}=k \wedge v^{\prime} \neq v\right)$, i.e, when there is step contention. Clearly, if $p_{i}$ does not lose round $k$, then it takes a linear number of read and write operations.

Theorem 2 and the fact that the universal construction of Herlihy [1991] uses only reads and writes, in addition to consensus objects, immediately imply:

COROLlary 3. Every sequential type has a solo-fast implementation from registers and CES objects.

\subsection{Time and Space Lower Bounds}

In this section, we prove time and space lower bounds for solo-fast implementations, showing that the non-constant step contention-free complexity and space complexity of our implementations are unavoidable.

One may be tempted to think that the linear lower bounds on the space and step complexity of solo-fast implementations follow from the results of Jayanti et al. [2000]. This is not true due to the following reason: their results are obtained by constructing executions in which processes continue executing their operations, using reads and writes only, even after they encounter step contention. Contrary to that, solo-fast implementations are allowed to apply stronger synchronization primitives in the face of step contention.

Our lower bounds hold for implementations of perturbable objects, a wide class of objects defined next; the following definition is equivalent to Definition 3.1 of Jayanti et al. [2000], when restricted to consider only deterministic implementations.

DEFINITION 3. An object $\mathcal{O}$ is perturbable if there is an operation instance op $p_{n}$ by process $p_{n}$, such that for any $p_{n}$-free execution $\alpha \lambda$ where no process applies more than a single event in $\lambda$ and for some process $p_{l} \neq p_{n}$ that applies no event in $\lambda$, there is an extension of $\alpha, \gamma$, consisting of events by $p_{l}$, such that $p_{n}$ returns 
different responses when performing op ${ }_{n}$ by itself after $\alpha \lambda$ and after $\alpha \gamma \lambda$. We say that $o p_{n}$ witnesses the perturbation of $\mathcal{O}$.

The following technical definition is required for our proofs. Note that if a process is active in the configuration resulting from a finite execution $\alpha$, then the process has exactly one enabled event, i.e., the event the process is about to apply in the configuration. If a process is idle but has begun a new operation-instance, then the first event of that operation-instance is enabled; otherwise, it has no enabled event.

Definition 4. A base object $o$ is covered after an execution $\alpha$ if the set $F$ of all the primitives applied to $o$ in $\alpha$ is historyless, and there is a process $p_{n}$ that has, after $\alpha$, an enabled event $e$ about to apply a nontrivial primitive from $F$ to o. We also say that $e$ covers $o$ after $\alpha$.

An execution $\alpha$ is $k$-covering if:

$-\alpha$ is step-contention free,

- there exists a set of processes $\left\{p_{j_{1}}, \ldots, p_{j_{k}}\right\}$ that does not contain process $p_{n}$, such that all the events of $\alpha$ are applied by processes in this set and each of the processes in the set has an enabled nontrivial event that covers a distinct base object after $\alpha$.

We call the set $\left\{p_{j_{1}}, \ldots, p_{j_{k}}\right\}$ a covering set of $\alpha$.

The second condition in Definition 4 implies that if an implementation has a $k$-covering execution, then its space complexity is at least $k$. We now prove a linear lower bound on the space complexity of any obstruction-free solo-fast implementation.

THEOREM 4. Let $A$ be an n-process solo-fast obstruction-free implementation of a perturbable object $\mathcal{O}$. The space complexity of $A$ is at least $n-1$.

Proof. Since $A$ is solo-fast, there exists a historyless set of primitives $S$ such that any process $p$ can apply only primitives from $S$ in executions that are stepcontention free for $p$.

Let $o p_{n}$ be the operation instance that witnesses the perturbation of $\mathcal{O}$. We prove the theorem by showing that $A$ has an $(n-1)$-covering execution.

The proof goes by induction. The empty execution is vacuously a 0 -covering execution. Assume that $\alpha_{i}$, for $i<n-1$, is an $i$-covering execution with covering set $\left\{p_{j_{1}}, \ldots, p_{j_{i}}\right\}$. Let $\lambda_{i}$ be the execution fragment that consists of the nontrivial events by processes $p_{j_{1}} \ldots p_{j_{i}}$ that are enabled after $\alpha_{i}$, arranged in some arbitrary order.

By Definition 3, there is an execution fragment $\gamma$ by some process $p_{j_{i+1}} \notin$ $\left\{p_{n}, p_{j_{1}}, \ldots, p_{j_{i}}\right\}$ such that $o p_{n}$ returns different responses after executions $\alpha_{i} \lambda_{i}$ and $\alpha_{i} \gamma \lambda_{i}$. We claim that $\gamma$ contains a nontrivial event that accesses a base object not covered after $\alpha_{i}$. Assume otherwise to obtain a contradiction. Since all events in executions $\alpha_{i} \lambda_{i}$ and $\alpha_{i} \gamma \lambda_{i}$ apply primitives from a historyless set, every nontrivial primitive applied to a base object in $\gamma$ is overwritten by some event in $\lambda_{i}$. Thus, the values of all base objects are the same after $\alpha_{i} \lambda_{i}$ and after $\alpha_{i} \gamma \lambda_{i}$. This implies that $o p_{n}$ must return the same response after both $\alpha_{i} \lambda_{i}$ and $\alpha_{i} \gamma \lambda_{i}$, which is a contradiction.

Journal of the ACM, Vol. V, No. N, April 2009. 
We extend $\alpha_{i}$ by letting $p_{j_{i+1}}$ execute the shortest prefix of $\gamma$ at the end of which it has an enabled nontrivial event about to access an object $o$ not covered after $\alpha_{i}$. We denote this prefix of $\gamma$ by $\gamma^{\prime}$. We define $\alpha_{i+1}$ to be $\alpha_{i} \gamma^{\prime}$. Thus, at the end of $\alpha_{i+1}, p_{j_{i+1}}$ has an enabled nontrivial event that accesses $o$. As none of the processes $p_{j_{1}}, \ldots p_{j_{i}}$ apply events in $\gamma^{\prime}$, we have that $\alpha_{i+1}$ is a step-contention free execution, after which processes $p_{j_{1}}, \ldots p_{j_{i+1}}$ have enabled events that cover distinct objects. Hence $\alpha_{i+1}$ is an $(i+1)$-covering execution. It follows that $A$ has an $(n-1)$-covering execution.

Next we prove a logarithmic lower bound on the step contention-free complexity of solo-fast implementations of perturbable objects. As the proof is quite involved, we first provide an informal description of its technique and structure.

Our goal is to construct a scenario in which process $p_{n}$ has to access a large number of base objects as it runs solo while performing an operation. To that end, our proof constructs longer and longer $r$-covering executions. The construction proceeds in phases. After each phase $r$ of the construction, we consider the path that $p_{n}$ will take if it runs solo after we 'unfreeze' the pending covering events (but we don't actually unfreeze these events). We denote this path by $\pi_{r}$. Note that some of the objects along this path may already be covered after phase $r$.

To construct phase $r+1$, we deploy a 'free' process, $p_{j_{r+1}}$, and let it run solo. As processes can only apply primitives from a historyless set, and as the implemented object is perturbable, we know that $p_{j_{r+1}}$ will eventually be about to write to an uncovered object, $O$, along $\pi_{r}$. This, however, may have the undesirable effect (from the perspective of an adversary) of making $\pi_{r+1}$ shorter than $\pi_{r}: p_{n}$ may read the information written by $p_{j_{r+1}}$ to $O$ (if we unfreeze its pending covering event) and not access some other objects farther along $\pi_{r}$ !

Note, however, that objects that are part of $\pi_{r}$ will be absent from $\pi_{r+1}$ only if $O$ precedes them in $\pi_{r}$. Thus the set of objects along $\pi_{r+1}$ that are covered (after phase $r+1$ ) is 'closer', in a sense, to the beginning of the path. It follows that if there are many phases $r$ such that $\left|\pi_{r}\right|$ decreases, then one of the paths $\pi_{r}$ must be 'long'.

To capture this intuition, we define $\Psi$, a monotonically-increasing progress function of the phase numbers. $\Psi_{r}$ is a $(\log n)$-digit binary number defined as follows. Bit 0 (the most significant bit) of $\Psi_{r}$ is 1 if and only if the first object in $\pi_{r}$ is covered; bit 1 of $\Psi_{r}$ is 1 if and only if the second object in $\pi_{r}$ exists and is covered, and so on. Note that we do not need to consider paths that are longer than $\log _{2} n$. If such a path exists, the lower bound clearly holds.

As mentioned before, to construct phase $r+1$, we deploy a free process, $p_{j_{r+1}}$, and let it run solo until it is about to write to an uncovered object, $O$, along $\pi_{r}$. In terms of $\Psi$, this implies that the covering event of $p_{j_{r+1}}$ might flip some of the digits of $\Psi_{r}$ from 1 to 0 . But $O$ corresponds to a more significant digit, and this digit is flipped from 0 to 1 , hence $\Psi_{r+1}>\Psi_{r}$ must hold. As we have $n-1$ processes to deploy, $\Psi_{r}$ must increase $n-1$ times and eventually it equals $n-1$. When it does, the length of $\pi_{r}$ is exactly $\log _{2} n$. The formal proof follows.

THEOREM 5. Let $A$ be an n-process solo-fast obstruction-free implementation of a perturbable object $\mathcal{O}$. A has a step-contention free execution in which a process accesses at least $\log _{2} n$ distinct base objects in the course of performing a single 
operation instance.

Proof. Since $A$ is solo-fast, there exists a historyless set of primitives $S$ such that any process $p$ can apply only primitives from $S$ in executions that are stepcontention free for $p$.

If there is an execution in which a process accesses more than $\log _{2} n$ distinct base objects in the course of performing a single operation instance in a step-contention free manner then we are done. Assume otherwise. We construct a step-contention free execution in which a process accesses exactly $\log _{2} n$ distinct base objects in the course of performing a single operation instance.

The construction proceeds in at most $n$ phases. In phase $r \geq 0$, we construct an execution $\alpha_{r} \delta_{r} \phi_{r}$ with the following structure:

$-\alpha_{r}$ is an $r$-covering execution with a covering set $p_{j_{1}}, \ldots, p_{j_{r}}$,

-in $\delta_{r}$, each of the processes $p_{j_{1}}, \ldots, p_{j_{r}}$ applies a nontrivial event to an object that is covered after $\alpha_{r}$, and

-in $\phi_{r}$, process $p_{n}$ runs solo after $\alpha_{r} \delta_{r}$ until it completes the operation instance $o p_{n}$.

Let $C\left(\alpha_{r}\right)$ denote the set of base objects that are covered after $\alpha_{r}$. Let $\pi_{r}=$ $O_{r}^{1} \ldots O_{r}^{i_{r}}$ denote the sequence of all distinct base objects accessed by $p_{n}$ in $\phi_{r}$ (after $\alpha_{r} \delta_{r}$ ) indexed according to the order in which they are first accessed by $p_{n}$. Also let $S_{\pi_{r}}$ denote the set of these base objects.

In execution $\alpha_{r} \delta_{r} \phi_{r}, p_{n}$ accesses $i_{r}$ distinct base objects. Thus, it suffices for the proof to construct such an execution with $i_{r}=\log _{2} n$. For $j \in\left\{1, \ldots, i_{r}\right\}$, we let $b_{r}^{j}$ be the indicator variable whose value is 1 if $O_{r}^{j} \in C\left(\alpha_{r}\right)$ and 0 otherwise. We associate an integral progress parameter, $\Psi_{r}$, with each phase $r \geq 0$, defined as follows:

$$
\Psi_{r}=\sum_{j=1}^{i_{r}} b_{r}^{j} \cdot 2^{\log _{2} n-j} .
$$

As we assume that $i_{r} \leq \log _{2} n$ for all $r, \Psi_{r}$ can be viewed as a $\log _{2} n$-digit number in base 2 whose $j$ 'th most significant bit is 1 if the $j$ 'th object in $\pi_{r}$ exists and is in $C\left(\alpha_{r}\right)$, or 0 otherwise. This implies that the number of base objects in $\pi_{r}$ that are covered after $\alpha_{r}$ equals the number of 1-bits in $\Psi_{r}$.

We now describe our construction. Let $\alpha_{0}$ and $\delta_{0}$ denote the empty execution; let $\phi_{0}$ denote the solo execution that results when $p_{n}$ performs the operation instance $o p_{n}$ starting from an initial configuration, and let $i_{0}$ denote the number of distinct objects accessed in $\phi_{0}$. Since $C\left(\alpha_{0}\right)=\emptyset$, we have $\Psi_{0}=0$. Suppose that, for some $r, 0 \leq r<n-1$, we have constructed $\alpha_{r} \delta_{r} \phi_{r}$ and $\Psi_{r}<n-1$.

As $\mathcal{O}$ is perturbable with operation instance $o p_{n}$ witnessing that, there is an execution fragment $\gamma_{r+1}$ by some process $p_{j_{r+1}} \notin\left\{p_{n}, p_{j_{1}}, \ldots, p_{j_{r}}\right\}$ such that $o p_{n}$ returns different responses to $p_{n}$ after executions $\alpha_{r} \delta_{r}$ and $\alpha_{r} \gamma_{r+1} \delta_{r}$. We claim that, in $\gamma_{r+1}, p_{j_{r+1}}$ applies a nontrivial event to an object in $S_{\pi_{r}} \backslash C\left(\alpha_{r}\right)$. Assume that $\gamma_{r+1}$ contains no nontrivial events to objects in $S_{\pi_{r}} \backslash C\left(\alpha_{i}\right)$ to obtain a contradiction. As $\alpha_{r} \gamma_{r+1}$ is step-contention free, all the events of $\gamma_{r+1}$ either access base objects not in $S_{\pi_{r}}$ or are overwritten by the events of $\delta_{r}$. It follows that $\alpha_{r} \gamma_{r+1} \delta_{r} \phi_{r}$ is also an execution of $A$ and that $\alpha_{r} \delta_{r} \phi_{r}$ and $\alpha_{r} \gamma_{r+1} \delta_{r} \phi_{r}$ are indistinguishable to $p_{n}$. 
This implies that $o p_{n}$ must return the same responses after both executions, which is a contradiction.

Let $\gamma_{r+1}^{\prime}$ be the shortest prefix of $\gamma_{r+1}$ after which $p_{j_{r+1}}$ has an enabled event, $e$, about to apply a nontrivial event to a base object $O_{r}^{k} \in S_{\pi_{r}} \backslash C\left(\alpha_{r}\right)$. Define $\alpha_{r+1}=\alpha_{r} \gamma_{r+1}^{\prime}, \delta_{r+1}=\delta_{r} e$ and let $\phi_{r+1}$ denote the execution fragment in which $p_{n}$ applies events by itself after $\alpha_{r+1} \delta_{r+1}$ as it performs the operation instance $o p_{n}$ to completion. It is easily verified that $\alpha_{r+1}$ is an $(r+1)$-covering execution and that $C\left(\alpha_{r+1}\right)=C\left(\alpha_{r}\right) \cup O_{r}^{k}$.

We claim that $\Psi_{r+1}>\Psi_{r}$ holds. As $O_{r}^{k} \notin C\left(\alpha_{r}\right)$, we have $b_{r}^{k}=0$. As the values of objects $O_{r}^{1} \cdots O_{r}^{k-1}$ are the same after $\alpha_{r} \delta_{r}$ and $\alpha_{r+1} \delta_{r+1}$, it follows that $b_{r}^{j}=b_{r+1}^{j}$ for $j \in\{1, \ldots, k-1\}$. This implies, in turn, that $O_{r}^{k}=O_{r+1}^{k}$. As $O_{r+1}^{k} \in C\left(\alpha_{r+1}\right)$, we have $b_{r+1}^{k}=1$. We get:

$$
\begin{aligned}
\Psi_{r+1}= & \sum_{j=1}^{i_{r+1}} b_{r+1}^{j} \cdot 2^{\log _{2} n-j} \\
= & \sum_{j=1}^{k-1} b_{r+1}^{j} \cdot 2^{\log _{2} n-j}+2^{\log _{2} n-k}+ \\
& \sum_{j=k+1}^{i_{+1}} b_{r+1}^{j} \cdot 2^{\log _{2} n-j} \\
= & \sum_{j=1}^{k-1} b_{r}^{j} \cdot 2^{\log _{2} n-j}+2^{\log _{2} n-k}+ \\
& \sum_{j=k+1}^{l_{r+1}} b_{r+1}^{j} \cdot 2^{\log _{2} n-j} \\
\geq & \sum_{j=1}^{k-1} b_{r}^{j} \cdot 2^{\log _{2} n-j}+2^{\log _{2} n-k} \\
> & \sum_{j=1}^{k-1} b_{r}^{j} \cdot 2^{\log _{2} n-j}+\sum_{j=k+1}^{i_{r}} b_{r}^{j} \cdot 2^{\log _{2} n-j} \\
= & \Psi_{r} .
\end{aligned}
$$

By definition, we have $0 \leq \Psi_{r} \leq n-1$ for all $r$. Furthermore, just a single process joins the execution in each phase. As we have shown that $\Psi$ is monotonically increasing with $r$, this implies that we eventually reach a phase $r^{*}$ with $\Psi_{r^{*}}=n-1$, i.e., $i_{r^{*}}=\log _{2} n$.

\section{OBSTRUCTION-FREE IMPLEMENTATIONS USING ARBITRARY PRIMITIVES}

In the previous sections, we considered obstruction-free implementations that can only apply synchronization primitives from a restricted set - either in all executions or just in step-contention free executions; the metric that we used counted the worst-case number of steps made by a process in step-contention free executions. However, the number of steps performed is not the only factor that contributes to the time complexity of concurrent objects. In practice, the performance of concurrent objects is influenced by the extent to which multiple processes access widely-shared memory locations simultaneously.

In 1993, Dwork et al. [1997] introduced a formal model to capture the phenomenon of memory contention in shared memory machines. Their model takes into consideration both the number of steps taken by a process and the number of stalls it incurs as a result of memory contention with other processes. In this section, we investigate obstruction-free implementations that can use arbitrary primitives even in step contention free executions. We analyze the worst-case stepand stall-complexities of these implementations. The definition of stalls that we use is similar to that used by Hendler and Shavit [2008] and Fich et al. [2005]. It is stricter than that used by Dwork et al. [1997] since we only count memory stalls caused by contention in writing, whereas Dwork et al. [1997] also count mem- 
ory stalls due to contention in reading. This definition captures the fact that when multiple processes apply nontrivial primitive operations simultaneously to the same base object, these operations are being serialized.

DeFINITION 5. Let $e$ be an event applied by a process $p$ to base object $r$, as it performs an operation instance $\Phi$ in execution $\alpha$. Let $\alpha=\alpha_{0} e_{1} \cdots e_{k} e \alpha_{1}$, where $\alpha_{0}$ and $\alpha_{1}$ are execution fragments and $e_{1} \cdots e_{k}$ is a maximal sequence of $k \geq 1$ consecutive nontrivial events, by distinct processes other than $p$, that access $r$. Then we say that $\Phi$ incurs $k$ memory stalls in $\alpha$ on account of $e$. The number of memory stalls incurred by $\Phi$ in $\alpha$ is the sum of memory stalls $\Phi$ incurs in $\alpha$ over all the events of $\Phi$ in $\alpha$.

Let $p$ be a process and consider the set of executions $\mathcal{E}_{p}$ that are indistinguishable to $p$ from an execution that is step-contention free to $p$. This includes all the executions that are step-contention free to $p$. From obstruction freedom, $p$ must make progress in any execution of $\mathcal{E}_{p}$. Let $\alpha \in \mathcal{E}_{p}$ be an execution and let $e$ be an event of $p$ that is enabled after $\alpha$. We say that $e$ is issued while $p$ is unaware of step contention. It might be that $p$ becomes aware of step contention when it receives the response of $e$. Nevertheless, the delay incurred by $p$ until it becomes aware of step contention includes the delay it incurs on account of $e$.

In a similar manner, we let $\mathcal{E}$ denote the set of executions that are indistinguishable to all processes from a step-contention free execution. Let $\alpha \in \mathcal{E}$ be an execution and let $e$ be an event that is enabled after $\alpha$. We say that $e$ is issued while no process is aware of step contention.

In the proofs that follow we consider the worst-case time complexity incurred by processes on account of the events they issue while being unaware of step contention.

\subsection{Binary Consensus and Perturbable Objects}

In this section we consider obstruction-free implementations of binary consensus and perturbable objects. We prove a tradeoff between the worst case operation step-complexity and stall-complexity of these implementations. More specifically, we prove that each such implementation has an execution in which no process is aware of step contention, such that either a process accesses at least $\sqrt{n}$ distinct base objects in the course of performing a single operation or some process incurs at least $\sqrt{n}$ stalls on account of a single event. This bound holds for all obstructionfree implementation of these objects, regardless of how processes behave when they encounter step contention. It implies that, for these implementations, a process can be made to incur a delay of length $\Omega(\sqrt{n})$ before any process becomes aware of step contention.

A one-shot binary consensus object restricts the argument to the propose operation to be in the domain $\{0,1\}$; each process calls propose at most once.

TheOREM 6. Let $A$ be an n-process obstruction-free implementation of binary consensus. There is an execution $\alpha$ of $A$ in which no process is aware of step contention and a process $p$ such that either $p$ accesses at least $\sqrt{n}$ distinct base objects in $\alpha$ or it incurs at least $\sqrt{n}$ stalls on account of an event it applies in $\alpha$.

Proof. Consider executions of $A$ in which processes $p_{1}, \ldots, p_{n-1}$ invoke propose with input 0 and process $p_{n}$ invokes propose with input 1 . Let $\phi$ be the execution

Journal of the ACM, Vol. V, No. N, April 2009. 
in which, starting from the initial configuration, $p_{n}$ performs its propose instance to completion. Let $B$ denote the set of base objects that are accessed in $\phi$. If $|B| \geq \sqrt{n}$ then we are done.

Otherwise, we construct a $p_{n}$-free execution at the end of which there is a subset of processes $S \subset\left\{p_{1}, \cdots, p_{n-1}\right\}$ of size exactly $\sqrt{n}$, all the processes of which have enabled nontrivial events about to access the same object in $B$. The execution is constructed inductively in at most $n-1$ phases. We denote the execution constructed in phases $1, \cdots, i$ by $\alpha_{i}$. Our construction maintains the following invariants for all $i \leq n-1$ :

$-\alpha_{i}$ is step-contention free,

—all the events of $\alpha_{i}$ are applied by processes in $\left\{p_{1}, \ldots, p_{i}\right\}$,

$-\alpha_{i}$ does not contain any nontrivial event applied to an object in $B$, and

—each of the processes $p_{1}, \cdots, p_{i}$ covers a base object in $B$ after $\alpha_{i}$.

We let $\alpha_{0}$ denote the empty execution. It is easily verified that the above invariants are vacuously met by $\alpha_{0}$. Assume we have constructed $\alpha_{i}$, for $i<n-1$, and that the number of enabled nontrivial events about to access any single object in $B$ at the end of $\alpha_{i}$ is less than $\sqrt{n}$. We now describe the construction of $\alpha_{i+1}$. We let process $p_{i+1}$ perform its instance of propose by itself after $\alpha_{i}$ until it either has an enabled nontrivial event about to access an object in $B$, or its propose instance completes.

We show that the latter cannot occur, by way of contradiction. The sequential specification of the consensus object imply that $p_{n}$ 's instance of propose returns 1 in $\phi$. Let $\sigma_{i+1}$ be the execution in which $p_{i+1}$ performs its propose instance after $\alpha_{i}$ until it completes. As $\alpha_{i} \sigma_{i+1}$ is $p_{n}$-free, the propose operation of $p_{i+1}$ returns 0 in $\alpha_{i} \sigma_{i+1}$.

By the induction hypothesis on $\alpha_{i}$, and as we assume that no nontrivial event was applied to an object in $B$ in $\sigma_{i+1}, \alpha_{i} \sigma_{i+1} \phi$ is an execution that is indistinguishable from $\phi$ to $p_{n}$. It follows that the responses of the instances of propose by $p_{i+1}$ and $p_{n}$ in $\alpha_{i} \sigma_{i+1} \phi$ are 0 and 1 , respectively. This contradicts the requirement that all processes decide on the same value.

Thus, at the end of $\alpha_{i+1}$, process $p_{i+1}$ has an enabled nontrivial event about to access a base object in $B$. By the induction hypothesis on $\alpha_{i}$, we get that at the end of $\alpha_{i+1}$, each of $p_{1}, \cdots, p_{i+1}$ has an enabled nontrivial event about to access an object in $B$, and that $\alpha_{i+1}$ is a step-contention free execution that contains no nontrivial event applied to an object in $B$.

As $|B|<\sqrt{n}$, there is a phase $j, j \leq n-1$, such that after $\alpha_{j}$ there exist at least $\sqrt{n}$ processes, all of which have enabled nontrivial events about to access the same object $o \in B$. Let $\alpha$ be some ordering of these events. Also let $\beta$ be the longest prefix of $\phi$ that does not access $o$, and let $e$ be $p_{n}$ 's enabled event after $\beta$. Then $p_{n}$ incurs at least $\sqrt{n}$ memory stalls in $\alpha_{j} \beta \alpha e$. To conclude the proof, we note that $\alpha_{j} \beta$ is step-contention free and that each of the events in $\alpha e$ is by a different process. Thus all the events of $\alpha_{j} \beta \alpha e$ are issued while no process is aware of step contention.

The proof of the following theorem follows the lines of that of Theorem 6 . 
THEOREM 7. Let $A$ be an n-process obstruction-free implementation of a perturbable object. There is an execution $\alpha$ of $A$ in which no process is aware of step contention and a process $p$ such that either $p$ accesses at least $\sqrt{n}$ distinct base objects in $\alpha$ or it incurs at least $\sqrt{n}$ stalls on account of an event it applies in $\alpha$.

Proof. Let $o p_{n}$ be the operation instance that witnesses the perturbation of $\mathcal{O}$. Let $\phi$ be the execution of $A$ in which, starting from the initial configuration, $p_{n}$ performs $o p_{n}$ until it completes it. Let $B$ denote the set of base objects that are accessed in $\phi$. If $|B| \geq \sqrt{n}$ then we are done. Assume otherwise.

We construct a $p_{n}$-free execution at the end of which there is a subset of processes $S \subset\left\{p_{1}, \cdots, p_{n-1}\right\}$ of size exactly $\sqrt{n}$, all the processes of which have enabled nontrivial events about to access the same object in $B$. The execution is constructed inductively in at most $n-1$ phases. We denote the execution constructed in phases $1, \ldots, i$ by $\alpha_{i}$. Our construction maintains the following invariants for all $i \leq n-1$ :

$-\alpha_{i}$ is step-contention free,

$-\alpha_{i}$ does not contain any nontrivial event applied to an object in $B$, and

- there exists a set of processes $\left\{p_{j_{1}}, \ldots, p_{j_{i}}\right\}$ that does not contain $p_{n}$, such that

- each of these processes has an enabled nontrivial event about to access a base object in $B$ after $\alpha$, and

—none of the events of $\alpha_{i}$ are applied by processes not in $\left\{p_{j_{1}}, \ldots, p_{j_{i}}\right\}$.

We let $\alpha_{0}$ denote the empty execution. It is easily verified that the above invariants are vacuously met by $\alpha_{0}$. Assume we have constructed $\alpha_{i}$, for $i<n-1$, and that the number of enabled nontrivial events about to access any single object in $B$ at the end of $\alpha_{i}$ is less than $\sqrt{n}$. We now describe the construction of $\alpha_{i+1}$. By the induction hypothesis on $\alpha_{i}$, no process has applied a nontrivial event in $\alpha_{i}$ to an object in $B$.

Let $\delta$ denote the execution fragment that consists of the events by $\left\{p_{j_{1}}, \ldots, p_{j_{i}}\right\}$ that are enabled after $\alpha_{i}$. As $\mathcal{O}$ is perturbable with operation instance $o p_{n}$ witnessing that, there is an execution fragment $\gamma$ by some process $p_{j_{i+1}} \notin\left\{p_{n}, p_{j_{1}}, \ldots, p_{j_{i}}\right\}$ such that $o p_{n}$ returns different responses to $p_{n}$ after executions $\alpha_{i} \delta$ and $\alpha_{i} \gamma \delta$. We claim that $p_{j_{i+1}}$ applies in $\gamma$ a nontrivial event to an object in $B$. Assume otherwise to obtain a contradiction. Then from the induction hypothesis and our assumption, $\alpha_{i} \gamma \delta \phi$ is an execution that is indistinguishable to $p_{n}$ from $\alpha_{i} \delta \phi$. It follows that the responses of $o p_{n}$ are the same in $\alpha_{i} \gamma \delta \phi$ and $\alpha_{i} \delta \phi$. This is a contradiction.

Let $\gamma^{\prime}$ be the shortest prefix of $\gamma$ after which $p_{j_{i+1}}$ has an enabled nontrivial event about to access a base object in $B$. We let $\alpha_{i+1}$ be $\alpha_{i} \gamma^{\prime}$. Thus, from the induction hypothesis applied to $\alpha_{i}$, we get that at the end of $\alpha_{i+1}$ each of $p_{j_{1}}, \cdots, p_{j_{i+1}}$ has an enabled nontrivial event about to access an object in $B$, and that $\alpha_{i+1}$ is a stepcontention free execution that contains no nontrivial event applied to an object in $B$.

As $|B|<\sqrt{n}$, there is a phase $k, k \leq n-1$, such that after $\alpha_{k}$ there exist at least $\sqrt{n}$ processes, all of which have enabled nontrivial events about to access the same object $o \in B$.

Let $\alpha$ be some ordering of these events. Also let $\beta$ be the longest prefix of $\phi$ that does not access $o$, and let $e$ be $p_{n}$ 's enabled event after $\beta$. Then $p_{n}$ incurs at least $\sqrt{n}$ memory stalls in $\alpha_{k} \beta \alpha e$ on account of $e$. To conclude the proof, we note that Journal of the ACM, Vol. V, No. N, April 2009. 
$\alpha_{k} \beta \alpha e$ and $\alpha_{k} \alpha \beta e$ are indistinguishable to $p_{n}$ and that $\alpha_{k} \alpha \beta e$ is step-contention free for $p_{n}$. Thus all the events of $\alpha_{k} \beta \alpha e$ are issued while no process is aware of step contention.

Theorems 6 and 7 establish the existence of executions in which a single operation incurs a tradeoff between step-complexity and stall-complexity. It is easy to construct executions in which the tradeoffs of Theorems 6 and 7 hold for multiple operations. This can be done by simply extending the executions constructed by these theorems until a quiescent configuration is reached and then repeating the construction.

\subsection{A Linear Lower Bound for Non-Failing Implementations}

We say that an obstruction-free implementation is non-failing if processes are not allowed to fail their operations when they become aware of step contention. For such implementations we obtain a lower bound that is stronger than the one obtained in Section 5.1.

Fich et al. [2005] prove a lower bound of $n-1$ on the worst-case number of stalls incurred by a process as it performs a single operation instance. This bound holds for non-failing obstruction-free implementations of objects in a class $\mathcal{G}$, that includes counter and single-writer snapshot objects. It can be shown that the same lower bound holds for any perturbable object. In the following, we prove that this bound holds in an execution in which all the events of the process whose operation instance incurs the linear complexity are issued while it is not aware of step contention.

The following definition of $k$-stall-execution is taken from [Fich et al. 2005] with minor terminology adaptation.

Definition 6. An execution $\alpha \sigma_{1} \cdots \sigma_{i}$ is a $k$-stall execution for process $p$ if

$-\alpha$ is p-free,

-there are distinct base objects $O_{1}, \ldots, O_{i}$ and disjoint sets of processes $S_{1}, \ldots, S_{i}$ whose union does not include $p$ and has size $k$ such that, for $j=1, \ldots, i$,

- each process in $S_{j}$ has an enabled nontrivial event about to access $O_{j}$ after $\alpha$, and

-in $\sigma_{j}$, process $p$ applies events by itself until it is first about to apply an event to $O_{j}$, then each of the processes in $S_{j}$ applies an event that accesses $O_{j}$, and, finally, $p$ applies an event that accesses $O_{j}$,

- all processes not in $S_{1} \cup \cdots \cup S_{i}$ are idle after $\alpha$,

- $p$ starts at most one operation instance in $\sigma_{1} \cdots \sigma_{i}$, and

-in every $\left(\{p\} \cup S_{1} \cup \cdots \cup S_{i}\right)$-free extension of $\alpha$, no process applies a nontrivial event to any base object accessed in $\sigma_{1} \cdots \sigma_{i}$.

In a $k$-stall execution for process $p, p$ incurs $k$ stalls, since it incurs $\left|S_{j}\right|$ stalls when it applies its first event to $O_{j}$, for $j=1, \ldots i$. The results of Fich et al. [2005] are obtained by proving that non-failing obstruction-free implementations of objects such as those mentioned above have $n-1$ stall executions for any process. Our contribution lies in the following technical lemma. It shows that a process $p$ is not aware of step contention in a $k$-stall execution for $p$. 
Lemma 8. Let $\alpha$ be a $k$-stall execution for process $p$. Then all of $p$ 's events in $\alpha$ are issued while $p$ is unaware of step contention.

Proof. Let $\alpha \sigma_{1} \ldots \sigma_{i}$ be a $k$-stall execution for process $p$ for some $k>0$. For $j=1, \ldots, i$, let $S_{j}$ and $O_{j}$ be as in Definition 6. For an execution $\sigma$, let $\sigma \mid \bar{p}$ be the subsequence of events in $\sigma$ that are applied by processes other than $p$.

We prove that the sequence of events $\theta=\alpha\left(\sigma_{1} \mid \bar{p}\right) \cdots\left(\sigma_{i} \mid \bar{p}\right)\left(\sigma_{1} \mid p\right) \cdots\left(\sigma_{i} \mid p\right)$ is an execution and that it is indistinguishable from $\alpha \sigma_{1} \ldots \sigma_{i}$ to all processes. Since $\theta$ is step-contention free for $p$, this will establish that all of $p$ 's events in $\alpha \sigma_{1} \ldots \sigma_{i}$ are issued while $p$ is unaware of step contention.

The proof goes by double induction. For $l=0, \ldots, i$, let $\alpha_{l}=\alpha \sigma_{0} \ldots \sigma_{l}$. The outer induction is on the executions $\alpha_{l}$. We prove that, for $l=0, \ldots, i$, $\theta_{l}=\alpha\left(\sigma_{1} \mid \bar{p}\right) \cdots\left(\sigma_{l} \mid \bar{p}\right)\left(\sigma_{1} \mid p\right) \cdots\left(\sigma_{l} \mid p\right)$ is an execution that is indistinguishable to all processes from $\alpha_{l}$. The claim holds vacuously for $l=0$. For $l<i$, assume that $\theta_{l}$ is an execution that is indistinguishable from $\alpha_{l}$ to all processes.

Consider the sequence of events $\sigma_{l+1} \mid \bar{p}$. By Definition 6, these events are enabled at the end of $\alpha_{l}$. Consequently, from outer induction hypothesis, they are also enabled at the end of $\theta_{l}$. As all the events of $\left(\sigma_{1} \mid p\right) \cdots\left(\sigma_{l} \mid p\right)$ are applied by $p$, the events of $\sigma_{l+1} \mid \bar{p}$ are enabled at the end of $\alpha\left(\sigma_{1} \mid \bar{p}\right) \cdots\left(\sigma_{l} \mid \bar{p}\right)$. Additionally, as each of the events of $\sigma_{l+1} \mid \bar{p}$ is applied by a distinct process in $S_{l+1}, \alpha\left(\sigma_{1} \mid \bar{p}\right) \cdots\left(\sigma_{l+1} \mid \bar{p}\right)$ is an execution.

By the induction hypothesis, all processes in $S_{1} \cup \cdots \cup S_{l}$ apply the same events and get the same responses in $\alpha\left(\sigma_{1} \mid \bar{p}\right) \cdots\left(\sigma_{l} \mid \bar{p}\right)$ and $\alpha_{l}$. As all the events of $\sigma_{l+1} \mid \bar{p}$ access $O_{l+1}$ and none of the events of $\sigma_{1} \cdots \sigma_{l}$ accesses $O_{l+1}$, it follows that all processes in $S_{1} \cup \cdots \cup S_{l+1}$ apply the same events and get the same responses in $\alpha_{l+1}$ and in $\alpha\left(\sigma_{1} \mid \bar{p}\right) \cdots\left(\sigma_{l+1} \mid \bar{p}\right)$, and hence also in $\theta_{l+1}$.

We next show that $\theta_{l+1}$ is an execution and that $p$ gets the same responses from the events it applies in it as in $\alpha_{l+1}$. We show this by inner induction on the number of events, $m$, applied by $p$ in $\left(\sigma_{1} \mid p\right) \cdots\left(\sigma_{l+1} \mid p\right)$.

The claim is obvious for $m=0$. Assume that $\left(\sigma_{1} \mid p\right) \cdots\left(\sigma_{l+1} \mid p\right)$ consists of $m>0$ events and that the claim holds for the first $m-1$ events. Let $e$ be the $m$ 'th event. Two cases exist. If $e$ accesses a base object $O \notin\left\{O_{1}, \ldots, O_{l+1}\right\}$, then, from Definition $6, O$ is not accessed in $\alpha \sigma_{1} \cdots \sigma_{l+1}$ by any process other than $p$. Thus, from the inner induction hypothesis, $O$ has the same value when $e$ accesses it in both $\alpha_{l}$ and $\theta_{l}$. Otherwise, suppose that $O=O_{j}$ for some $j \in\{1, \ldots, l+1\}$. The subsequence of events that precede $e$ in accessing $O_{j}$ is $\left(\sigma_{j} \mid \bar{p}\right)$ in both $\alpha_{l+1}$ and $\theta_{l+1}$. Consequently, from inner and outer induction hypotheses, $O$ has the same value when accessed by $e$ in both $\alpha_{l+1}$ and $\theta_{l+1}$. It follows that, in both cases, $e$ returns the same response in $\alpha_{l}$. Hence also $p$ applies the same events, and gets the same responses from these events, in both $\alpha_{l}$ and $\theta_{l}$.

As all processes apply the same events, and get the same responses from these events in both $\alpha_{l}$ and $\theta_{l}$, and as $\alpha_{l}$ is an execution, it follows that $\theta_{l}$ is also an execution. This concludes the proof of the lemma.

The proof of Theorem 6 of Fich et al. [2005] can be used to establish that any non-failing obstruction-free $n$-process implementation of a perturbable object has an $(n-1)$-stall execution for any process that shares the implementation. Combining that with Lemma 8 gives the following.

Journal of the ACM, Vol. V, No. N, April 2009. 
THEOREM 9. Let $A$ be an n-process non-failing obstruction-free implementation of a perturbable object. Then for any process $p$ there is an execution $\alpha$ of $A$ such that $p$ incurs in $\alpha$ at least $n-1$ stalls on account of events that it issues while it is unaware of step contention, as it performs a single operation instance.

Theorem 9 constructs an execution in which a single operation incurs a linear number of stalls. An executions in which this complexity is incurred by multiple operations can be constructed by simply extending the execution constructed by the theorem until a quiescent configuration is reached and then repeating the construction.

\subsection{Lock-Based Implementations}

The time-complexity results we presented in this section establish that, for any obstruction-free implementation of a large class of objects, a single operation may incur a delay of length $\Omega(\sqrt{n})$ in an execution in which no process is aware of step contention, regardless of how processes proceed when they do become aware of step contention. Moreover, if processes are not allowed to fail their operations even in the face of step contention, then any process can be made to incur a delay of at least $n-1$ stalls on account of an event it issues while not being aware of step contention.

A natural question that arises is whether lock-based implementations of such objects exist that are not subject to these lower bounds. In other words, is there a separation in terms of worst-case operation time complexity between obstructionfree and lock-based implementations? We now show that this is indeed the case. Consider an implementation of a perturbable or binary consensus object that is guarded by a lock; that is, only the process that holds the lock can invoke an operation on the object. The lock can be implemented by using a binary tournament-tree implementation of mutual exclusion, employing read and write operations, such as the tournament tree algorithm due to Peterson and Fischer [1977]. The operations of capturing the lock and releasing it access $O(\log n)$ distinct base objects and incur $O(\log n)$ stalls. Hence there are lock-based implementations that do not incur the complexities of Theorems 6,7 and 9 .

One may argue that this complexity separation is artificial, since processes may perform an unbounded number of steps while they wait for a lock. This is only true, however, if processes busy-wait. If processes signal each other by using semaphores [Dijkstra 2002] or condition variables [Lea 1999] instead of employing busy-waiting, then they can indeed avoid the high worst-case complexities incurred by obstruction-free implementations. ${ }^{2}$

\section{DISCUSSION}

This paper studies obstruction-free implementations by defining their specification, presenting generic object implementations, and proving lower bounds on their complexity. Our lower bounds concentrate on the cost in uncontended executions (which

${ }^{2}$ Whether or not semaphores should be used instead of busy-waiting in practice obviously depends on system parameters, such as the actual level of contention for the lock and the context-switch time.

Journal of the ACM, Vol. V, No. N, April 2009. 
are argued to be the most frequent in practice); some of them do not restrict the behavior of the processes in contended situations where processes might be using locks, randomization or other expensive mechanisms. By measuring the complexity of the weakest form of implementations that do not use locks known to date, our results capture the seemingly inherent cost of avoiding locking.

We have established tight bounds on the cost of generic obstruction-free implementations. There might be more efficient obstruction-free solutions for specific problems. For obstruction-free consensus, for example, an $\Omega(\sqrt{n})$ lower bound on the number of registers (or historyless objects) was proved by Fich et al. [1998]. There is however a gap since the upper bound is $O(n)$; moreover, these results do not bound the step contention-free complexity of obstruction-free consensus. It would be interesting to close the gap between these upper bounds and the logarithmic lower bound we prove for the step contention-free complexity of any generic solo-fast implementation.

Luchangco et al. [2003] presented a generic object implementation that uses only constant number of reads and writes when an operation runs in the absence of point contention. Unfortunately, in their implementation this also means lack of pending operations; moreover, an operation invoked after a prefix with point contention may have to apply $C \& S$ primitives, even if it does not encounter any point contention itself. In this paper, we gave a generic solo-fast implementation. Our solo-fast implementation performs $O(n)$ reads and writes, even in the absence of step contention. By employing adaptive collect [Afek et al. 1999; Attiya and Fouren 2003], the step complexity can be made to depend only on the point contention, so it is constant when there is no point contention. By employing adaptive collect for unbounded concurrency [Gafni et al. 2001], it can be made independent of the number of processes.

Aguilera et al. [2006] proposed the concept of abortable objects that are, at first glance, similar to our obstruction-free objects. Abortable objects are live: operations on them return either matching responses or a predefined $\perp$ value. To prevent trivial implementations and ensure some level of progress in face of failures, they satisfy an additional non-triviality requirement that $\perp$ can only be returned in the presence of interval contention, and in the eventual absence of step contention, repeated invocations may return $\perp$ only finitely many times. As a result, a suspended operation may prevent a concurrent operation from making progress only for a bounded period of time. Aguilera et al. [2006] argue that if $\perp$ is allowed to be output only in the presence of step contention, then the resulting implementations may not preserve correctness under composition: by replacing base object of a correct (linearizable, live and non-trivial) implementation with its correct implementation from some finer base objects, we may not obtain a correct implementation from the finer base objects. As we show in the appendix, composability can be achieved at the expense of a slightly stronger definition of obstruction-freedom based on step contention. We propose a definition of a live object that is only allowed to return $\perp$ in the presence of step contention with an additional guarantee that an operation may only take effect within the interval of one of its occurrences. We also provide a generic implementation of such objects from read-write objects.

Journal of the ACM, Vol. V, No. N, April 2009. 


\section{ACKNOWLEDGMENTS}

We would like to thank Partha Dutta, Ron Levy, and Eric Ruppert for important discussions on the topic of this paper, and Eshcar Hillel and the referees for helpful comments on an earlier draft.

\section{REFERENCES}

Afek, Y., Stupp, G., And Touitou, D. 1999. Long-lived adaptive collect with applications. In Proceedings of the 40th Annual Symposium on Foundations of Computer Science (FOCS). IEEE Computer Society, Washington, DC, USA, 262-272.

Afek, Y., Stupp, G., And Touitou, D. 2002. Long-lived adaptive splitter and applications. Distributed Computing 15, 2, 67-86.

Aguilera, M. K. And FrøLund, S. 2003. Strict linearizability and the power of aborting. Tech. Rep. HPL-2003-241, HP Laboratories Palo Alto. Dec.

Aguilera, M. K., Frolund, S., Hadzilacos, V., Horn, S. L., And Toueg, S. 2006. Abortable shared objects. In Proceedings of the 20th International Symposium on Distributed Computing (DISC). Springer-Verlag, Berlin / Heidelberg, 534-536.

Aspnes, J. ANd Herlihy, M. 1990. Fast randomized consensus using shared memory. J. Algorithms 11, 3, 441-461.

Attiya, H. And Fouren, A. 2003. Algorithms adapting to point contention. J. ACM 50, 4, $444-468$.

Attiya, H., Guerraoui, R., Henlder, D., and Kouznetsov, P. 2006. Synchronizing without locks is inherently expensive. In Proceedings of the 25th Annual ACM Symposium on Principles of Distributed Computing (PODC). ACM, New York, NY, USA, $300-307$.

Attiya, H., Guerraoui, R., And Kouznetsov, P. 2005. Computing with reads and writes in the absence of step contention. In Proceedings of the 19th International Symposium on Distributed Computing (DISC). Springer-Verlag, Berlin / Heidelberg, 122-136.

Attiya, H. And Welch, J. L. 2004. Distributed Computing: Fundamentals, Simulations and Advanced Topics, 2nd ed. John Wiley \& Sons, Hoboken, New Jersey.

Boichat, R., Dutta, P., Frølund, S., and Guerraoui, R. 2003. Deconstructing Paxos. ACM SIGACT News Distributed Computing Column 34, 1 (March), 47 - 67. Revised version of EPFL Technical Report 200106, January 2001.

Dijkstra, E. W. 2002. Cooperating sequential processes. In The origin of concurrent programming: from semaphores to remote procedure calls. Springer-Verlag New York, Inc., New York, NY, USA, 65-138.

Dwork, C., Herlihy, M., and WaArts, O. 1997. Contention in shared memory algorithms. Journal of the ACM 44, 6, 779-805.

Fich, F., Herlihy, M., And Shavit, N. 1998. On the space complexity of randomized synchronization. J. ACM 45, 5, 843-862.

Fich, F. E., Hendler, D., And Shavit, N. 2005. Linear lower bounds on real-world implementations of concurrent objects. In Proceedings of the 46th Annual Symposium on Foundations of Computer Science (FOCS). IEEE Computer Society, Washington, DC, USA, 165-173.

Gafni, E., Merritt, M., and Taubenfeld, G. 2001. The concurrency hierarchy, and algorithms for unbounded concurrency. In Proceedings of the 20th Annual ACM Symposium on Principles of Distributed Computing (PODC). ACM, New York, NY, USA, 161-169.

Hendler, D. And Shavit, N. 2008. Solo-valency and the cost of coordination. Distributed Computing 21, 1, 43-54.

Herlihy, M. 1991. Wait-free synchronization. ACM Transactions on Programming Languages and Systems 13, 1 (January), 124-149.

Herlihy, M., Luchangco, V., And Moir, M. 2003. Obstruction-free synchronization: Doubleended queues as an example. In Proceedings of the 23rd IEEE International Conference on Distributed Computing Systems (ICDCS). IEEE Computer Society, Washington, DC, USA, $522-529$. 
Herlihy, M., Luchangco, V., Moir, M., And Scherer III, W. N. 2003. Software transactional memory for dynamic-sized data structures. In Proceedings of the 22nd Annual ACM Symposium on Principles of Distributed Computing (PODC). ACM, New York, NY, USA, 92-101.

Herlihy, M. AND Wing, J. M. 1990. Linearizability: a correctness condition for concurrent objects. ACM Transactions on Programming Languages and Systems 12, 3 (June), 463-492.

Jayanti, P., Tan, K., And Toueg, S. 2000. Time and space lower bounds for nonblocking implementations. SIAM Journal on Computing 30, 2, 438-456.

LAMPoRT, L. 1998. The part-time parliament. ACM Transactions on Computer Systems 16, 2 (May), 133-169.

LEA, D. 1999. Concurrent Programming in Java(TM): Design Principles and Patterns, 2nd ed. Addison-Wesley, Boston, MA, USA.

Loui, M. C. And Abu-Amara, H. H. 1987. Memory Requirements for Agreement Among Unreliable Asynchronous Processes. JAI Press, Greenwich, Conn., 163-183.

LuchangCo, V., Moir, M., And Shavit, N. 2003. On the uncontended complexity of consensus. In Proceedings of the 17th International Symposium on Distributed Computing (DISC). SpringerVerlag, Berlin / Heidelberg, 45-59.

Peterson, G. L. AND Fischer, M. J. 1977. Economical solutions for the critical section problem in a distributed system (extended abstract). In Proceedings of the 9th annual ACM symposium on Theory of computing (STOC). ACM, New York, NY, USA, 91-97.

Yang, J., Neiger, G., And Gafni, E. 1998. Structured derivations of consensus algorithms for failure detectors. In Proceedings of the 17th Annual ACM Symposium on Principles of Distributed Computing (PODC). ACM, New York, NY, USA, 297-306.

\section{A. EXTENDED INTERFACE OF OBSTRUCTION-FREE OBJECTS}

In this section, we explore the possibility of enriching the interface of an obstructionfree implementation with an additional pause repsonse $\perp$. As we observed (Theorem 1), a consensus implementation from read-write registers cannot be made wait-free even if operations are allowed to return failure response in the face of step contention. Thus allowing an implementation to return a fail indication in the face of step contention does not guarantee wait-freedom in general. This may be inconvenient if we want every invocation to eventually return control to the client.

We therefore consider an extended interface between clients and implementations that guarantees that every invocation eventually returns. In the extended interface, each operation should either return a legal response for the invoked operation, or, if there is step contention, one of two special values $\perp$ and $\emptyset$. If the implementation is certain that the operation did not have an effect, $\emptyset$ is returned, indicating that the client is free to invoke any operation it wishes. Otherwise, a special pause value $\perp$ is returned, and the client must re-invoke the same operation until a non- $\perp$ response is received. To make sure that the implementation does not always fall back to outputting $\perp$, we require that $\perp$ can only be returned if the invoked operation can take effect in some extension of the current execution in which that operation is not re-invoked.

\section{A.1 Definitions}

We extend $R$, the set of responses of an object, with two special values: a pause value $\perp \notin R$ and a fail value $\emptyset \notin R$. The definition of a well-formed local history is extended to require that if an invocation $i$ is followed by the response $\perp$, then the subsequent event, if one exists, is $i$.

Journal of the ACM, Vol. V, No. N, April 2009. 
The definition of linearizability is further extended so that invocations returning fail are removed from the linearized history, while at most one of identical invocations returning pause is considered as one operation.

Specifically, consider an execution $\alpha$; the corresponding history is obtained as follows. For every invocation $i$ in $\alpha$ that is not followed by a response in $R$, we insert in $\alpha$ a response $\perp$ immediately after the last event of the process that invokes $i$ in $\alpha$. Now the history $H$ is defined as a subsequence of $\alpha$ that consists of invocation and response of high-level operations (including $\emptyset$ and $\perp$ responses). A local history of $\alpha$ is a subsequence of $H$ consisting of all events of some process $p$. We only consider executions $\alpha$ that induce well-formed local histories.

Let $\bar{H}$ be any local history of $\alpha$. Let $i$ be an invocation in $\bar{H}$ on an object $x$. A fragment of the form $i, r$ in $\bar{H}$, where $r \in R$, is called an occurrence of $i$ (returning $r)$. Since an invocation occurrence may return $\perp$ and be re-invoked later, there can be a number of occurrences of $i$ in a history. Consider the longest fragment of the form $i$ or $i, \perp, i, \ldots, \perp, i$ in $\bar{H}$. Recall that we completed every pending invocation in $\alpha$ with $\perp$, so each such fragment in $\bar{H}$ is followed by a matching response $r \in R$. If $r \notin\{\perp, \emptyset\}$, we call $i, r$ or $i, \perp, i \ldots, \perp, i, r$ a complete operation. If $r=\emptyset$, we call $i, \emptyset$ or $i, \perp, i \ldots, \perp, i, \emptyset$ a failed operation. If $r=\perp$, we call $i, \perp$ or $i, \perp, i, \ldots, \perp, i$ or $i, \perp, i \ldots, \perp, i, \perp$ a pending operation. Since $\bar{H}$ is well-formed, a pending operation is a suffix of $\bar{H}$ ( $\perp$ cannot be followed by an invocation other than $i$ ).

Now we say that a well-formed history $H$ is linearizable if there is a sequential history $H^{\prime}$ containing occurrences of all the complete operations in $H$ and a subset of occurrences of the pending operations in $H$, with at most one occurrence per operation, in which each $\perp$ is replaced with a matching response $r \in R-\{\emptyset, \perp\}$, such that $H^{\prime}$ is legal and it respects the order of non-concurrent operation occurrences in $H$. When taken in the context of the extended notions of complete and pending operations, a history is linearizable if we can assign linearization points to all complete operations and a subset of pending operations so that the linearization point assigned to an operation may only belong the interval of one of the operation's occurrences.

An implementation is linearizable if it produces only linearizable histories. An implementation is live if every invocation occurrence $i$ returns in a finite number of its own steps (although a value in $\{\perp, \emptyset\}$ can be returned). A linearizable implementation is valid if (1) an invocation occurrence returns $\perp$ only when it is not step-contention free, (2) an invocation occurrence $i$ returns $\emptyset$ only when the corresponding operation (the longest fragment of the form $i, \emptyset$ or $i, \perp, i, \ldots, \perp, i, \emptyset$ in the local history) is not step-contention free, and (3) if an invocation $i$ performed by $p_{i}$ returns $\perp$ at the end of an execution $\alpha$, then $i$ is nontrivial and there exists an execution $\alpha^{\prime}$ that agrees with $\alpha$ on the state of $p_{i}$, i. e., $\alpha\left|i=\alpha^{\prime}\right| i$, and $i$ appears in some linearization of $H\left(\alpha^{\prime}\right)$. The last property provides a non-triviality criterion on the use of pause responses: an invocation made by a process $p_{i}$ is allowed to return $\perp$ only if the corresponding operation takes effect in some indistinguishable (for $p_{i}$ ) execution. It is immediate that any live and valid implementation is obstruction-free.

Note that our definition ensures that in any execution of a linearizable implementation, every operation takes effect (if it does) within one of its occurrences, and, in 
particular, before it stops taking steps in that execution. As a result, if a process fails (stops taking steps) before completing its operation's occurrence, then the operation takes effect (if it does) before the process fails, making the implementations strictly linearizable [Aguilera and Frølund 2003].

\section{A.2 Generic Object Implementation}

This section presents a generic obstruction-free (live and valid) implementation of any type. Our generic construction extends the universal nonblocking implementation of Herlihy [1991] to handle pause and fail responses in the presence of step contention. By the validity requirement, an operation invoked by a process $p_{i}$ is only allowed to return $\perp$ if its invocation takes effect in some indistinguishable (for $p_{i}$ ) execution. This makes our extension of Herlihy's construction nontrivial: we need to carefully account for the cases in which $\perp$ cannot be returned.

As in Herlihy's construction, our implementation is built from consensus objects, which in our case are obstruction-free. ${ }^{3}$

The implementation (presented in Figure 4) follows a deterministic sequential implementation of an object type $T$. An object is represented as a linked list; an element of the list represents an operation applied to the object. The list of operations clearly determines the list of corresponding responses. A process makes an invocation by appending a new element to the end of the list. The algorithm assumes a function response(invs, inv) that returns the response matching the invocation $i n v$ in a sequential execution of invocations from list invs (under the condition that inv $\in$ invs).

We assume that invocations corresponding to different operations are uniquely identifiable. The algorithm uses the following shared variables:

- $n$ atomic single-writer, multi-reader registers $L_{1}, \ldots, L_{n}$. Process $p_{i}$ stores in $L_{i}$ its last view of the object state in the form of a linked list of operations that $p_{i}$ observed to have been applied on the object.

- $C[]$ is an unbounded array of obstruction-free (linearizable, live and valid) consensus objects. The array is used to agree on the order in which invocations are put into the linked list of operations $L_{1}, \ldots, L_{n}$.

Note that we do not assume that lines of the pseudo-code presented in Figure 4 are executed atomically. Informally, the algorithm works as follows. When a process $p_{i}$ executes an invocation inv, it identifies the longest list $L_{j}$ (line 2). Let $k=\left|L_{j}\right|$ be the number of operations on the object witnessed by $p_{j}$. If $i n v$ is already in $L_{j}$, the response associated with inv in $L_{j}$ is returned (line 5). This ensures that inv takes effect at most once, even if repeated several times. If it is not the first instance of inv, and $k>\left|L_{i}\right|$ (i.e., inv did not "win" any OF Consensus to which it was proposed), then $p_{i}$ returns $\emptyset$ (line 9). Otherwise, $p_{i}$ proposes inv to $C[k+1]$ (line 10). If $C[k+1]$ returns $\perp$ (which can happen only if step contention is detected), then $p_{i}$ returns $\perp$ (line 14). If the propose operation (a) returns $\emptyset$, or (b) returns a non-inv response and the current invocation occurrenceis not the first occurrence of $i n v$ (i.e., if repeated is true), then $p_{i}$ returns $\emptyset$ (line 19). The condition


Paxos-style consensus algorithms, appears in Appendix B.2.

Journal of the ACM, Vol. V, No. N, April 2009. 
repeated is true in (b) is needed since, otherwise, a non- $\{i n v, \perp, \emptyset\}$ invocation could have been returned by $C[k]$ in the absence of step contention.

If $C[k+1]$ returns $i n v$, then $p_{i}$ returns the response associated with inv (line 23). Otherwise, the procedure is repeated, now at position $k+2$. If $C[k+2]$ returns a non- $\{i n v, \perp\}$ response, then $p_{i}$ returns $\emptyset$ (line 36). The second consensus operation ensures that the implementation is valid, namely, that $(1) \perp$ is never returned if the corresponding invocation instance is step-contention free, $(2) \emptyset$ is never returned if the corresponding operation is step-contention free, and (3) if $\emptyset$ is never returned if $i$ is nontrivial and there exists an execution $\alpha^{\prime}$ that agrees with $\alpha$ on the state of $p_{i}$, i.e., $\alpha\left|i=\alpha^{\prime}\right| i$, and $i$ appears in some linearization of $H\left(\alpha^{\prime}\right)$.

This algorithm implies the next theorem:

THEOREM 10. Every sequential type $T$ has a linearizable, live and valid implementation from registers.

Proof. We immediately observe that the implementation in Figure 4 is live: it contains no blocking statements and the underlying obstruction-free consensus objects are live. We prove now that it is valid and linearizable.

Let $\alpha$ be any well-formed finite execution of the implementation in Figure 4. First, we complete each pending invocation $i$ in $\alpha$ with a $\perp$ response put immediately after the last step of the process that invoked $i$. Now every invocation $i$ in $\alpha$ is followed by a matching response.

We say that an invocation $i n v$ is fixed at index $k$ in $\alpha$, and we write fixed $(\alpha, i n v, k)$, if some propose operation on $C[k]$ returns inv in $\alpha$ (in line 10 or 24). Since each $C[k]$ is a consensus object, at most one invocation can be fixed at every index. We say that index $k$ is decided in $\alpha$ if there is an invocation $i_{k}$ such that fixed $\left(\alpha, i_{k}, k\right)$. Let $k^{*}$ be the highest decided index in $\alpha$. Since a process only accesses an OF consensus object $C[k+1]$ if some process previously obtained a non- $\{\perp, \emptyset\}$ from $C[k]$, we have the following property.

. P1 Each $k=1, \ldots, k^{*}$ is decided.

Since each $C[k]$ is a consensus implementation, it is allowed to return at most one non- $\{\perp, \emptyset\}$ value in $\alpha$. Thus, for each $k=1, \ldots, k^{*}$, we can define $r_{k}$ as the response returned by $i_{k}$ in a sequential order of invocations $i_{1}, i_{2}, \ldots, i_{k}$ (according to the sequential specification of $T$ ). Hence, we have the following property:

. P2 For each $k=1, \ldots, k^{*}$, if $i_{k}$ returns a non- $\{\perp, \emptyset\}$ response, then the response is $r_{k}$.

Now we show that $\alpha$ includes no occurrence $i_{k}, r\left(k=1, \ldots, k^{*}\right)$ where $r=\emptyset$. Indeed, $i_{k}$ returns $\emptyset$ (i) in lines 9, 19 or 19 only if $i_{k}$ is not fixed in any OF consensus object to which it was proposed so far (verified in lines 3 and A.2, or line A.2 or line A.2, respectively), (ii)in lines 16 or 30 only if $i_{k}$ is trivial and object $C[k]$ or $C[k+1]$ returns $\perp$ (lines A.2 and A.2 or A.2 and A.2, respectively). Thus, given $\mathrm{P} 2$, a fixed invocation can only return $\perp$ or $r_{k}$ in $\alpha$.

Recall that each $C[k]$ is linearizable. For each $k=1, \ldots, k^{*}$, some invocation $C[k]$.propose $\left(i_{k}\right)$ takes effect, i.e., $C[k]$.propose $\left(i_{k}\right)$ is included in every linearization of the execution on $C[k]$. Thus, for each $k=1, \ldots, k^{*}$, we can determine $o_{k}$ be the 


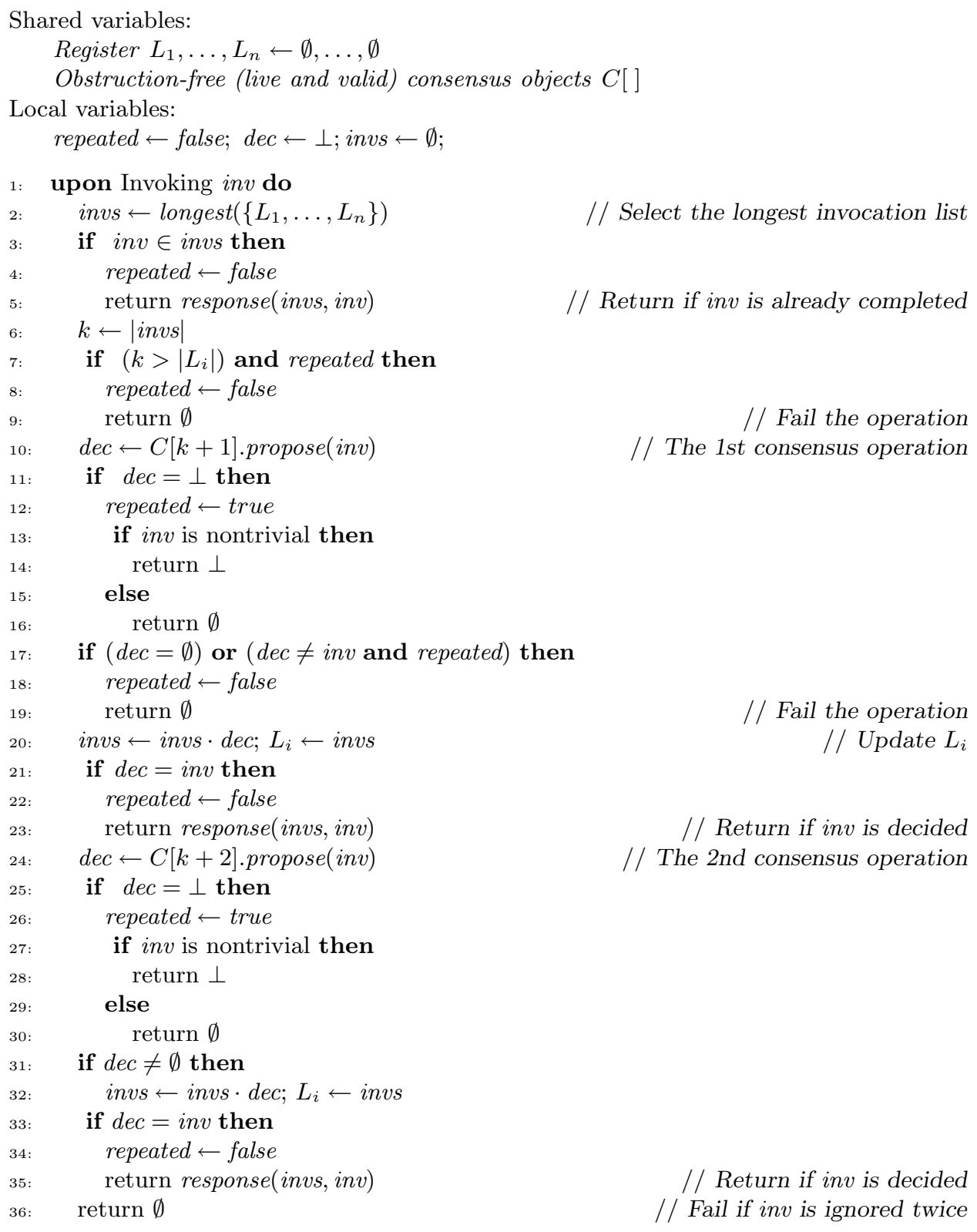

Fig. 4. An obstruction-free implementation of $T$ : code for process $p_{i}$ 
occurrence $i_{k}, r$ which includes the invocation $C[k]$.propose $\left(i_{k}\right)$ that takes effect in $\alpha$.

Now we construct a sequential history $H^{\prime}=o_{1}^{\prime}, o_{2}^{\prime}, \ldots, o_{k^{*}}^{\prime}$ where for each $k=$ $1, \ldots, k^{*}, o_{k}^{\prime}=i_{k}, r_{k}$. Note that $H^{\prime}$ contains no invocation that returned $\emptyset$ in $\alpha$, every invocation that returned $r \notin\{\perp, \emptyset\}$, and a subset of invocations that returned $\perp$.

By construction $H^{\prime}$ is legal. To show that $\alpha$ is linearizable, it sufficient to prove that $H^{\prime}$ preserves the order of non-concurrent occurrences $o_{1}, \ldots, o_{k}$ in $\alpha$.

Indeed, suppose that $o_{\ell}$ precedes $o_{m}$ in $\alpha$. Since the indexes of $o_{k} \mathrm{~s}$ in $H^{\prime}$ are monotonically increasing it is enough to show that $\ell<k$. Suppose, by contradiction, that ell $\geq m$. Since all $i_{k} \mathrm{~s}\left(k=1, \ldots, k^{*}\right)$ are distinct,ell $>m$. By definition, $o_{\ell}$ and $o_{m}$ contain the linearized invocations $C[\ell]$.propose $\left(i_{\ell}\right)$ and $C[m]$.propose $\left(i_{m}\right)$, respectively. By the algorithm (lines 2 and 20), a process $p_{j}$ may only access $C[\ell]$ if all $k<\ell$ are already fixed, i.e., the process previously made sure that for some $L_{j} \in\left\{L_{1}, \ldots, L_{n}\right\},\left|L_{j}\right|=\ell-1$. But since $C[m]$ is linearizable, it could not return $r_{m}$ before it was linearized, i.e., before the beginning of the occurrence $o_{m}$. Thus, $m$ is not fixed in the shortest prefix of $\alpha$ that includes the invocation of $o_{\ell}-\mathrm{a}$ contradiction.

Finally, we prove that our implementation is valid. Assume that inv returns a value in $\{\perp, \emptyset\}$. We have the following cases:

(1) $\perp$ is returned in line 14 or line 28 . Thus, a propose operation on $C[k+1]$ or $C[k+2]$ returned $\perp$. This can only happen if the current occurrence of $i n v$ is not step contention-free, nontrivial and there was a concurrent propose operation on $C[k+1]$ or $C[k+2]$. Since each $C\left[k^{\prime}\right], k^{\prime} \in \mathbb{N}$, is valid, there is an execution $\alpha^{\prime}$ such that $p_{i}$ cannot distinguish the prefix of $\alpha$ up to returning $\perp$ and $\alpha^{\prime}$, and fixed $\left(\alpha^{\prime}\right.$, inv,$\left.k+1\right)$ or fixed $\left(\alpha^{\prime}\right.$, inv,$\left.k+2\right)$. Thus, inv takes effect in $\alpha^{\prime}$ and $\alpha^{\prime}$ is indistinguishable from the shortest prefix of $\alpha$ in which inv returns $\perp$.

(2) $\emptyset$ is returned in line 9 . This can only happen when there were at least one prior occurrence of $i n v$ and all previous occurrences of $i n v$ returned $\perp$. Thus, the corresponding operation is not step contention-free.

Note that if $p_{i}$ reaches line 10 while repeated is true, then inv is not fixed at any $k^{\prime} \leq k$ and the last propose $(i n v)$ invoked by $p_{i}$ on $C[k+1]$ returned $\perp$.

(3) $\emptyset$ is returned in line 16 or line 30 . This can only happen if inv is trivial. Also, similarly to case (2) above, the current invocation of of inv is not stepcontention free.

(4) $\emptyset$ is returned in line 19. That is, either $C[k+1]$ returned $\emptyset$, or inv was not fixed at any $k^{\prime} \leq k+1$. Thus, inv did not take effect, and the corresponding operation was not step-contention free.

(5) $\emptyset$ is returned in line 36. That is, inv is not fixed at any index less than $k+2$ and $C[k+2]$ returned either $\emptyset$ or a value $\operatorname{dec} \notin\{\perp, \emptyset, i n v\}$. In the former case, since the corresponding propose $(i n v)$ operation is allowed to return $\emptyset$ only if it is not step-contention free and it did not take effect, the current instance of inv is not step-contention free and it did not take effect either.

Now assume that $C[k+2]$ returned $\operatorname{dec} \notin\{\perp, \emptyset, i n v\}$ and let $p_{j}$ be the process that previously proposed dec to $C[k+2]$. By the algorithm, before proposing, 
$p_{j}$ has made sure that for some $p_{l} \in \Pi,\left|L_{l}\right|=k+1$ (lines 2, 6 and 20). But the longest list in $\left\{L_{1}, \ldots, L_{n}\right\}$ seen by $p_{i}$ in the beginning of the current instance of inv had length $k$ (line 6). Thus, $p_{l}$ took steps in the interval of the current instance of $i n v$, i.e., the instance is not step-contention free. Moreover, inv was not fixed at any $k^{\prime} \leq k+2$, and thus did not take effect.

Thus, the implementation is linearizable, live, and valid.

\section{B. CONSENSUS IMPLEMENTATIONS}

In this section, we give two simple implementations of obstruction-free consensus in the read-write shared memory model: one for the standard interface presented in Section 3, and one for the extended interface introduced in Appendix A.

\section{B.1 Obstruction-Free Consensus}

As suggested by Herlihy et al. [2003], an obstruction-free consensus algorithm can be derived by "de-randomizing" a randomized consensus algorithm [Aspnes and Herlihy 1990]. Figure 5 depicts a simple implementation of consensus from registers that is allowed to block or return fail in case of step contention. For simplicity, we assume that processes propose distinct values. (This is the way consensus is used in our paper.)

Our implementation is based on the adopt-commit protocol of Yang et al. [1998]. The one-shot adopt-commit abstraction accepts a value $v \in V$ as an argument and returns a special abort value (abort $\notin V)$, or a pair $\left(c, v^{\prime}\right)$, where $c$ is a boolean and $v^{\prime} \in V$. If $\left(c, v^{\prime}\right)$ is returned and $c=$ false, we say that $v^{\prime}$ is adopted. If $\left(c, v^{\prime}\right)$ is returned and $c=$ true, we say that $v^{\prime}$ is committed. The abstraction guarantees that (i) every adopted or committed value is an input value of some process; (ii) no two processes commit on or adopt different values, and (iii) if all inputs are the same, then no process aborts or adopts a value (every process that returns must commit). Also, it is easy to observe that the adopt-commit protocol proposed by Yang et al. [1998] guarantees an additional property: if a process proposes a value $v$ and obtains abort, then no other process can adopt or commit $v$.

In the algorithm, every process executes repeated instances of the adopt-commit protocol with monotonically increasing indexes, accepting all adopted values as its decision estimates, until some value is committed or abort is returned. Then the process returns the committed value or $\emptyset$ (in case an instance of adopt-commit returns abort).

Obviously in a step contention-free execution, eventually at most one value will be proposed to some instance of the adopt-commit protocol, and this value will be committed. On the other hand, if a value committed in some instance of adoptcommit, then no process can adopt or commit a different value. If abort is returned in instance $k$ of adopt-commit, then the proposed value was not committed in any instance $k^{\prime}<k$ and cannot be adopted in instance $k$.

\section{B.2 Obstruction-Free Consensus with Extended Interface}

Here we present a linearizable, live and valid consensus implementation. Interestingly, our algorithm (Figure 6 ) translates the long-lived $\diamond$ Register implementation [Boichat et al. 2003], from message-passing to read-write shared memory.

Journal of the ACM, Vol. V, No. N, April 2009. 


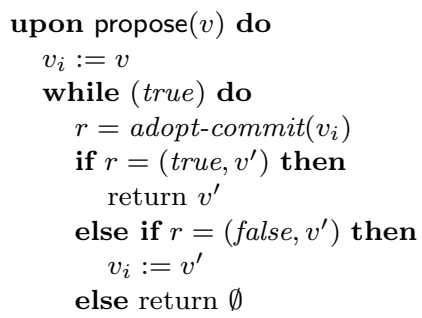

Fig. 5. Obstruction-free consensus: code for process $p_{i}$

Every process $p_{i}$ maintains a current estimate of the decision value of the consensus, denoted by $v_{i}$ and initialized to $\perp$, and two counters $r_{i}$ and $w_{i}$, both initialized to 0 . The counter $r_{i}$ denotes the round number adopted by the current operation, and $w_{i}$ denotes the last round number in which $p_{i}$ "announced" its estimate $v_{i}$. Each process $p_{i}$ is designated a single-writer multi-reader register $R_{i}$, initialized to $(0,0, \perp)$ that is written by $p_{i}$ and read by all processes. A boolean repeated, initialized to false, indicates whether this is not the first occurrence of the current operation.

Roughly, the algorithm can be decomposed into two phases. In the first phase, process $p_{i}$ chooses the highest unique round number, "registers" the round (writes in $R_{i}$, line 5 ), and collects the shared memory. In the second phase, $p_{i}$ adopts the value announced in the highest round (or its own proposal if no value is announced so far) as $v_{i}$, "announces" it with the current round number, and collects the shared memory again. If $p_{i}$ observes that some process registered a higher round (which can occur only when there is step contention) and a value different from $v_{i}$ is announced in a higher round (which can only happen if the value proposed by $p_{i}$ cannot be decided), then $p_{i}$ returns $\emptyset$ (line 16). If step contention is detected and $v_{i}$ is still the highest announced value, then $p_{i}$ returns $\perp$ (line 19). Otherwise, $p_{i}$ returns $v_{i}$. These two phases (register and announce) ensure that once $p_{i}$ returns $v_{i}$, no process will ever announce a value different from $v_{i}$ in a higher round. As a result, no two processes can return different non- $\{\perp, \emptyset\}$ values, no value in $\{\perp, \emptyset\}$ can be returned in the absence of step contention, $\emptyset$ is never returned if the corresponding operation took effect, and $\perp$ is never returned if there is an indistinguishable execution in which the operation did take effect.

THEOREM 11. There is a linearizable, live and valid consensus implementation from registers.

PROOF. The implementation in Figure 6 is clearly live, since there are no cycles or wait statements in the code.

Let $\alpha$ be any finite well-formed execution of the algorithm in which some non$\{\perp, \emptyset\}$ value $v$ is returned by some process. (If there is no such value, the execution is trivially linearizable.) To prove linearizability of the implementation, it is sufficient to show that (i) every returned non- $\{\perp, \emptyset\}$ value is a previously proposed value, and (ii) no two processes return different non- $\{\perp, \emptyset\}$ values.

We observe that (i) follows directly from the algorithm.

To prove (ii), we first show that if a process $p_{i}$ returns a non- $\{\perp, \emptyset\}$ value $v$ in a 




Fig. 6. Consensus implementation with extended interface: code for process $p_{i}$

round $r$, then no process can announce a value different from $v$ in a higher round. Indeed, let $p_{j}$ be the first process to announce a value $v^{\prime}$ in a round $r^{\prime}>r$. We immediately observe that $p_{j}$ registered $r^{\prime}$ (line 5) after $p_{i}$ announced $v$ in round $r$ (otherwise, $p_{i}$ would see that a round higher than $r$ is registered in line 13 and return $\perp$ or $\emptyset$ ). Thus, when $p_{j}$ collects the shared memory in round $r^{\prime}$ (line 6 ), $v$ is the value announced in the highest round number. Thus, $v=v^{\prime}$. Since before returning a non- $\{\perp, \emptyset\}$ value, every process announces the value, we have (ii).

We conclude by showing that the implementation is valid (recall the definition in Appendix A.1). Responses in $\{\perp, \emptyset\}$ are returned only if step contention is detected (line 13). Specifically, if $\emptyset$ is returned (line 16), then $p_{i}$ made sure that its current estimate is not the value announced in the highest round (line 14), so the estimate cannot be decided. That is, the propose $(v)$ operation of $p_{i}$ did not take effect in the execution. If $\perp$ is returned (line 19), then no process has previously announced a value different from $v_{i}$ in a higher round. Thus, every process returns $v_{i}$ in the extension of the current execution in which no process writes a value different from $v_{i}$ in $\left\{R_{j}\right\}$. 